\title{
Systematic effects in the measurement of polarization by the PLANCK telescope
}

\author{
G. Franco ${ }^{1}$, P. Fosalba ${ }^{1,2}$, and J. A. Tauber ${ }^{1}$ \\ 1 Research and Science Support Department of ESA, ESTEC, PO Box 299, 2200 AG Noordwijk, \\ The Netherlands \\ 2 Institut d'Astrophysique de Paris, 98bis boulevard Arago, 75014 Paris, France
}

Received 2 October 2002 / Accepted 6 March 2003

\begin{abstract}
We present estimates of the response to polarized signals by the PLANCK telescope. These estimates are based on a set of simulations, using a physical optics code (GRASP8), for linearly polarized detectors at different frequencies and located in different positions of an early design of the PLANCK focal plane. We show how the optical aberration introduced by the studied focal plane configuration affects absolute and relative orientation of the sky signals polarization planes. In addition, we compute the spurious signal introduced by the telescope optics into a PLANCK-type measurement of the sky polarization. Our analysis shows that the spurious polarization expected in a PLANCK-like experiment is typically of the order of $0.2 \%$ of the unpolarized intensity.
\end{abstract}

Key words. space vehicles: instruments - telescopes - polarization - cosmology: cosmic microwave background space vehicles

\section{Introduction}

The CMB temperature anisotropy carries an essential information on the origin and evolution of large scale structures in the universe. Its accurate measurement by experiments probing large (see e.g., Bennett et al. 1994) and small scales (see e.g., Scott et al. 2003 and references therein) already allows us to constrain cosmological models with good precision (see e.g., Lewis \& Bridle 2002). In addition, the quadrupole temperature anisotropy at the last scattering surface generates a polarization signal (of order $10 \%$ of the CMB intensity) carried by the scalar and tensor modes of the CMB anisotropy (Rees 1968). This polarized anisotropy is a unique probe of primordial gravitational waves (Crittenden et al. 1993a,b) and the reionization era of the universe (see e.g., Zaldarriaga 1997; $\mathrm{Hu}$ 2000). Moreover, CMB polarization helps break the degeneracy affecting the cosmological parameter estimation from CMB anisotropy data alone (e.g., Zaldarriaga et al. 1997).

Detecting CMB polarization is a serious experimental challenge which requires an unprecedented experimental sensitivity, good knowledge and clean removal of foreground contamination, and a careful analysis of systematic effects, the latter recently attracting increasing attention (see e.g., Carretti et al. 2001; Kaplan \& Delabrouille 2001; Leahy et al. 2001; Yurchenko 2001; Fosalba et al. 2002). Current upper limits for $\mathrm{CMB}$ polarizations are at the level of $10 \mu \mathrm{K}$ (Hedman et al. 2001; Keating et al. 2001; de Oliveira-Costa et al. 2003).

Send offprint requests to: P. Fosalba, e-mail: fosalba@iap. fr
Despite great experimental efforts, CMB polarization has remained undetected until very recently with the first detection of $E$-mode polarization by the DASI experiment (Kovac et al. 2002). A new generation of CMB experiments is being specifically designed to image the polarization anisotropies in the microwave sky with ground-based telescopes (e.g., COMPASS), interferometers (e.g., CBI), balloons (MAXIPOL, B2K), and satellites (MAP, PLANCK). In particular, the PLANCK experiment $^{1}$ will provide multi-frequency full-sky maps of the polarized sky with a sensitivity better than $5 \mu \mathrm{K}$ for practically all the channels (Tauber 2000). Ultimately, the polarization performance of PLANCK depends on the ability to minimize spurious instrumental effects which contaminate the sky signal.

In this paper, we investigate the impact of one specific instrumental systematic - the optical aberration in the PLANCK telescope - in the measurement of polarized signals. The PLANCK telescope is an off-axis aplanatic system with a very wide field of view of $\sim 8^{\circ}$ on the sky. The focal plane is filled with individual corrugated horns that collect radiation from the telescope and deliver it to band-limited detectors (with bandwidth $\sim 25 \%$ ) within the $30-850 \mathrm{GHz}$ frequency range. Detectors collect largely linearly polarized radiation, defined either by a waveguide hybrid discriminator or by a linear grid. This experimental scheme is common to other current CMB polarization experiments (e.g., MAP).

\footnotetext{
${ }^{1}$ PLANCK is the third medium-sized mission in the Horizons 2000 Scientific Program of the European Space Agency, http://astro.estec.esa.nl/Planck
} 
Although PLANCK was not originally devised as a $\mathrm{CMB}$ polarization imager, its current design incorporates 7 polarized channels between 30 and $353 \mathrm{GHz}$, in the Lowand High-frequency instruments (LFI and HFI hereafter), including a total of 88 linearly polarized detectors. PLANCK, to be launched in 2007, is currently in its Phase B (detailed design) during which many features, such as the focal plane layout, are being optimised for the polarization measurement. The scheme exploits the fact that many detectors in the focal plane are sensitive to the same frequency band and are spatially arranged such that their polarization planes are rotated with respect to each other by multiples of $45^{\circ}$. In this way, by combining samples from several linearly polarized detectors, it is possible to extract all the polarization information in terms of the Stokes parameters (see e.g., Kraus 1982; Couchot et al. 1999). However, an optimal measurement relies critically on the similarity of the polarized angular responses, also called beam or radiation patterns, of the differently oriented polarized horns. These radiation patterns will be difficult to measure accurately on the ground and they will have to be reconstructed in flight using celestial sources such as planets and the Galactic plane.

In this paper we shall study two basic issues regarding the ability of PLANCK to measure polarization:

- What is the effect of the feed and telescope optics on the properties of the measured polarized sky signal? In particular, are the absolute and relative orientation of polarization planes in the sky preserved by the optics?

- What is the impact of the instrumental optics on the differential measurement of polarization?

In what follows we shall use Physical Optics (PO) modeling to simulate the interaction of radiation with PLANCK telescope optical system. Unlike Geometrical optics (GO), which is characterized by the neglect of the wavelength, $\mathrm{PO}$ uses the concepts of electromagnetic transverse waves and their propagation properties to simulate the behaviour of light in optical systems, being the most accurate method to simulate diffraction, interference and polarization phenomena. In this study we use GRASP8, a software package developed by TICRA (1997), to compute the polarized radiation pattern on the sky for different transmitting feeds located in the focal plane ${ }^{2}$ (see Sect. A.1 for further details). In particular, we shall simulate the feeds located at three representative (off-axis) positions and at two different frequencies, and examine the effect of the optical asymmetries on the measurement of the Stokes parameters. Using such simulations we aim at answering the two basic questions mentioned above and draw some general conclusions regarding the impact of the PLANCK design under study in this paper, for the measurement of $\mathrm{CMB}$ polarization.

The paper is organized as follows: in Sect. 2 we address the problem of simulating and comparing the beam (or radiation) patterns generated using PO modeling. Results of the

\footnotetext{
${ }^{2}$ By the principle of reciprocity, modeling a feed transmitting from the focal plane to the sky is equivalent to modeling a detector horn, in the same position, collecting radiation from the sky, since, by time reversal, the transmission pattern is the same as the reception pattern.
}

simulations are presented and discussed in Sect. 3 and our final conclusions are given in Sect. 4. In the Appendix A we give a full description of the simulations procedures with GRASP8 code.

\section{Modeling far-field radiation patterns}

\subsection{Introducing reference frames}

Polarized radiation can be fully described by the projection of the electric field vector, $\boldsymbol{E}$, in a 2-dimensional orthogonal basis. For the far field radiation pattern, $\boldsymbol{E}_{\text {far }}$, we shall adopt Ludwig's 3rd definition of co- and cross-polarization (Ludwig 1973),

$\boldsymbol{E}_{\mathrm{far}}=E_{\mathrm{co}} \sigma_{\mathrm{co}}+E_{\mathrm{cr}} \sigma_{\mathrm{cr}}$

where $\sigma_{\mathrm{co}}$ and $\sigma_{\mathrm{cr}}$ are the co- and cross-polarization unit vectors which relate to the usual spherical polar basis $\left(\sigma_{\theta}, \sigma_{\phi}\right)$ as follows,

$\sigma_{\mathrm{co}}=\sigma_{\theta} \sin \phi+\sigma_{\phi} \cos \phi$

$\sigma_{\mathrm{cr}}=\sigma_{\theta} \cos \phi-\sigma_{\phi} \sin \phi$.

By projecting onto the Cartesian coordinate system,

$\sigma_{\theta}=\sigma_{x} \cos \theta \cos \phi+\sigma_{y} \cos \theta \sin \phi-\sigma_{z} \sin \theta$

$\sigma_{\phi}=-\sigma_{x} \sin \phi+\sigma_{y} \cos \phi$

one finds that at boresight, i.e., the north pole of the sphere $\left(\theta \rightarrow 0^{\circ}\right)$, it holds that $\sigma_{\mathrm{co}} \rightarrow \sigma_{y}$ and $\sigma_{\mathrm{cr}} \rightarrow \sigma_{x}$ (see Eqs. (2) and (3)), so the chosen polarization basis lies along the Cartesian one. Away from boresight, Ludwig's polarization basis is obtained by parallel-transporting the local Cartesian basis $\left(\sigma_{x}, \sigma_{y}\right)$ along great circles over the sphere.

\subsection{Defining cross-polarization for non-ideal antennas}

Here we shall define a robust way of characterizing crosspolarization in non-ideal optical systems. In particular, we discuss cross-polarization definitions for the case of feeds with arbitrary orientations in the focal plane.

Let us denote the orientation of a given feed (with respect to its symmetry or rotation axis) by $\psi_{\mathrm{FP}}$. We define a reference orientation, denoted by $\psi_{\mathrm{FP}}^{n r}=0^{\circ}$, with respect to which we can define any arbitrary orientation of a given feed. Similarly, we must introduce a corresponding far-field reference frame with respect to which the sky beam patterns from a given feed with an arbitrary orientation in the focal plane, will be referred to.

For an ideal telescope ${ }^{3}$ with an on-axis feed, one can always find a reference frame in the far field for which the crosspolarization power (see Eq. (1)) vanishes $\left(E_{\mathrm{cr}}^{2}=0\right)$. This constitutes a convenient reference frame for sky beam patterns of a given feed. However, for a real telescope, any asymmetry in the antenna system will generally introduce a non-vanishing cross-polarization component in the far-field radiation pattern. Moreover, non-ideal telescope optics also introduce a differential rotation of the principal plane of polarization in the far field with respect to that physically applied to the feed in the focal plane.

\footnotetext{
${ }^{3}$ We define an ideal telescope as one with rotationally symmetric reflectors and on-axis fully linearly polarized detectors.
} 
We recall that a proper measurement of polarization in terms of the Stokes parameters of the sky signal requires that the orientation of the feeds in the focal plane be such that the principal planes of polarization on the sky are spaced by $45^{\circ}$. Therefore, it is important to choose an appropriate method for determining this principal plane of polarization, which is here defined as that in which the Cross Polarization Discrimination (XPD) is maximized.

We define maximal cross-polar discrimination angle, $\phi_{\mathrm{XPD}}$, as the angle by which one should rotate the reference frame in the sky in order to maximize the Cross Polar Discrimination, XPD. Below we introduce three different methods for the computation of $\phi_{\mathrm{XPD}}$, each one relying on a different definition of $\mathrm{XPD}^{4}$ :

- The 1st Method is the most commonly used in the literature. $\phi_{\mathrm{XPD}}$ is the angle that maximizes XPD defined as the ratio of the (single) co-polar power peak to the highest cross-polarization power peak. In $\mathrm{dB}$ units we can write the maximized XPD as,

$$
\begin{aligned}
& \Delta W_{\max }=\Delta W\left(\phi_{\mathrm{XPD}}\right)=10 \log _{10}\left[\frac{\operatorname{Max}\left(E_{\mathrm{co}}^{\mathrm{rot}} E_{\mathrm{co}}^{\mathrm{rot} *}\right)}{\operatorname{Max}\left(E_{\mathrm{cr}}^{\mathrm{rot}} E_{\mathrm{cr}}^{\mathrm{rot} *}\right)}\right]_{\left(\phi=\phi_{\mathrm{XPD}}\right)} \\
& =10 \log _{10}\left[\operatorname{Max}\left(\left(E_{\mathrm{co}}^{\mathrm{rot}}\right)^{2}\right)\right]-10 \log _{10}\left[\operatorname{Max}\left(\left(E_{\mathrm{cr}}^{\mathrm{rot}}\right)^{2}\right)\right] .
\end{aligned}
$$

- In the 2nd Method, XPD is defined as the difference between the co-polar power peak and the cross-polarization power along the same direction. Since $\phi_{\mathrm{XPD}}$ is the angle that maximizes this difference, we can write

$$
\begin{aligned}
\Delta W_{\max }= & \Delta W\left(\phi_{\mathrm{XPD}}\right)=10 \log _{10}\left[\operatorname{Max}\left(\left(E_{\mathrm{co}}^{\mathrm{rot}}\right)^{2}\right)\right] \\
& -10 \log _{10}\left[\left(E_{\mathrm{cr}}^{\mathrm{rot}}\left(\theta_{\mathrm{Max}}, \phi_{\mathrm{Max}}\right)\right)^{2}\right]
\end{aligned}
$$

where $\theta_{\mathrm{Max}}$ and $\phi_{\mathrm{Max}}$ are the coordinates of the co-polar power peak in the spherical coordinate system $(\theta, \phi)$.

- The 3rd Method determines $\phi_{\mathrm{XPD}}$ as the angle that maximizes the difference between the integrated co-polar and cross-polarization amplitudes over the main beam, and thus the maximized XPD is given by

$$
\begin{aligned}
\Delta W_{\max }= & \Delta W\left(\phi_{\mathrm{XPD}}\right)=10 \log _{10}\left[\int_{-\theta_{0}}^{+\theta_{0}} \int_{0}^{\pi}\left(E_{\mathrm{co}}^{\mathrm{rot}}\right)^{2} \mathrm{~d} \Omega\right] \\
& -10 \log _{10}\left[\int_{-\theta_{0}}^{+\theta_{0}} \int_{0}^{\pi}\left(E_{\mathrm{cr}}^{\mathrm{rot}}\right)^{2} \mathrm{~d} \Omega\right]
\end{aligned}
$$

where the integration interval on $\theta,\left[-\theta_{0} ;+\theta_{0}\right]$, must be taken wide enough to encompass most of the power in the co- and cross-polarization patterns. We chose $\theta_{0}$ to be at least twice the Full Width at Half Maximum (FWHM) of the co-polar power pattern.

Note that in the equations above $E_{\mathrm{co}}^{\text {rot }}$ and $E_{\mathrm{cr}}^{\text {rot }}$ are the (complex) co- and cross-polarization components of the radiation pattern, after having performed a rotation of the far field coordinate system by an angle ( $\phi_{\mathrm{XPD}}$ in this case) with respect to

\footnotetext{
${ }^{4}$ For simplicity of notation, XPD will always be expressed in $\mathrm{dB}$ units as a power difference, $\Delta W$.
}

the original reference frame (i.e., the far-field frame defined for a non-rotated feed in the focal plane),

$E_{\mathrm{co}}^{\mathrm{rot}}=E_{\mathrm{cr}} \sin \phi_{\mathrm{XPD}}+E_{\mathrm{co}} \cos \phi_{\mathrm{XPD}}$

$E_{\mathrm{cr}}^{\mathrm{rot}}=E_{\mathrm{cr}} \cos \phi_{\mathrm{XPD}}-E_{\mathrm{co}} \sin \phi_{\mathrm{XPD}}$.

In general, the far-field radiation pattern of an asymmetric radiating system fed by a corrugated horn has a cross-polarization pattern with a multiple peak (rather than a simple peak) structure with several maxima which are not spatially coincident with nor symmetrically placed with respect to the co-polar peak (e.g. see Fig. A.4). Such an asymmetric cross-polarization pattern for non-ideal antennas makes the cross polar discrimination angle defined in the 1st method potentially ambiguous. In principle, the 3rd method should be appropriate for measurements of diffuse polarized emission, as it is based on integrating radiation filling the main beam (i.e., an extended region around boresight), while the 2nd method is more appropriate for measuring polarization from point sources as it relies on a local measurement of power radiated.

\subsection{Differential rotation of the polarization plane}

Let us consider again an ideal antenna system. In this case, it is expected that a rotation of a given feed around its own symmetry axis, from an initial position, $\psi_{\mathrm{FP}}^{i}$, to a final position, $\psi_{\mathrm{FP}}^{f}$, would result in an equal rotation of the principal plane of polarization of the radiation pattern in the sky ${ }^{5}$. Thus we can write,

$\phi_{\mathrm{XPD}}^{f}-\phi_{\mathrm{XPD}}^{i}=-\left(\psi_{\mathrm{FP}}^{f}-\psi_{\mathrm{FP}}^{i}\right)$.

Setting $\phi_{\mathrm{XPD}}^{i}=\phi_{\mathrm{XPD}}^{n r}$ as the maximal cross-polarization discrimination angle for the beam pattern when the feed is in its original or non-rotated position $\left(\psi_{\mathrm{FP}}^{i}=0^{\circ}\right)$, the previous equation becomes,

$\phi_{\mathrm{XPD}}-\phi_{\mathrm{XPD}}^{n r}=-\psi_{\mathrm{PP}}$.

This equation holds for any ideal antenna system if the polarization properties of the radiation pattern are equally preserved for all orientations of the feed in the focal plane.

However, for a non-ideal antenna system, such isotropy is broken and, due to factors such as the asymmetry of the reflectors or the off-axis positioning of the feed, Eq. (9) will only hold to a first-order approximation, i.e., $\phi_{\mathrm{XPD}}-\phi_{\mathrm{XPD}}^{n r} \approx-\psi_{\mathrm{PP}}$. Consequently, in order to quantify how good this approximation is (or on the contrary, how non-ideal is the antenna), we shall define the residual angle, $\Delta_{\phi}$, as the additional angle to which the reference frame of the beam pattern in the far-field should be rotated, in addition to $\left(-\psi_{\mathrm{FP}}\right)$, so as to have the copolar axis of the beam pattern reference frame aligned with the principal polarization direction. Thus we define

$\Delta_{\phi} \equiv \phi_{\mathrm{XPD}}-\phi_{\mathrm{XPD}}^{n r}-\left(-\psi_{\mathrm{FP}}\right)$

${ }^{5}$ In fact, in a dual reflector system such as that of PLANCK, the rotation of the principal plane of polarization on the sky would be symmetric and not equal to the rotation of the feed, due to the "mirroring" of the radiation pattern in the beam coordinate system when the signal is reflected from one mirror to the other. Equation (8) takes this symmetry into account. 
Table 1. Maximal cross-polarization discrimination angle $\phi_{\mathrm{XPD}}, \Delta W_{\max }$ and the residual angle $\Delta_{\phi}$ for the $30 \mathrm{GHz}$ and the $100 \mathrm{GHz}$ feeds on positions 1, 4 and 27, for different orientations $\psi_{\mathrm{FP}}$ of the feeds being $\phi_{\mathrm{XPD}}$ determined by the 1st Method described in Sect. 2.2 and $\Delta W_{\mathrm{Max}}$ evaluated using Eq. (4).

Results on the Determination of $\Delta_{\phi}$ by Maximizing the Difference

Between the Co- and Cross-polar Power Peaks (1st Method)

\begin{tabular}{|c|c|c|c|c|c|c|c|c|c|}
\hline \multirow{2}{*}{$\frac{30 \mathbf{G H z}}{\psi_{\mathrm{FP}}}$} & \multicolumn{3}{|c|}{ Position 1} & \multicolumn{3}{|c|}{ Position 4} & \multicolumn{3}{|c|}{ Position 27} \\
\hline & $\phi_{\mathrm{XPD}}$ & $\Delta W_{\text {Max }}$ & $\Delta_{\phi}$ & $\phi_{\mathrm{XPD}}$ & $\Delta W_{\text {Max }}$ & $\Delta_{\phi}$ & $\phi_{\mathrm{XPD}}$ & $\Delta W_{\text {Max }}$ & $\Delta_{\phi}$ \\
\hline$\left({ }^{\circ}\right)$ & $\left({ }^{\circ}\right)$ & $(\mathrm{dB})$ & $\left(^{\circ}\right)$ & $\left({ }^{\circ}\right)$ & $(\mathrm{dB})$ & $\left({ }^{\circ}\right)$ & $\left(^{\circ}\right)$ & $(\mathrm{dB})$ & $\left({ }^{\circ}\right)$ \\
\hline 0 & 0.4 & 33 & 0 & 4.8 & 29 & 0 & 7.9 & 26 & 0 \\
\hline 45 & -43.9 & 33 & 0.7 & -39.1 & 29 & 1.2 & -35.1 & 26 & 2 \\
\hline 60 & -58.9 & 33 & 0.7 & -54.0 & 29 & 1.2 & -49.7 & 26 & 2.4 \\
\hline 90 & -89.3 & 33 & 0.3 & -83.8 & 29 & 1.4 & -79.6 & 26 & 2.5 \\
\hline 120 & -119.9 & 33 & -0.3 & -114.2 & 29 & 1.0 & -110.8 & 26 & 1.3 \\
\hline 135 & -135.0 & 33 & -0.4 & -129.8 & 29 & 0.5 & -126.5 & 26 & 0.6 \\
\hline 180 & -179.6 & 33 & 0 & -175.2 & 29 & 0 & -172.1 & 26 & 0 \\
\hline
\end{tabular}

\begin{tabular}{|c|c|c|c|c|c|c|c|c|c|}
\hline \multirow{2}{*}{$\frac{100 \mathbf{~ G H z}}{\psi_{\mathrm{FP}}}$} & \multicolumn{3}{|c|}{ Position 1} & \multicolumn{3}{|c|}{ Position 4} & \multicolumn{3}{|c|}{ Position 27} \\
\hline & $\phi_{\mathrm{XPD}}$ & $\Delta W_{\operatorname{Max}}$ & $\Delta_{\phi}$ & $\phi_{\mathrm{XPD}}$ & $\Delta W_{\mathrm{Max}}$ & $\Delta_{\phi}$ & $\phi_{\mathrm{XPD}}$ & $\Delta W_{\mathrm{Max}}$ & $\Delta_{\phi}$ \\
\hline$\left({ }^{\circ}\right)$ & $\left({ }^{\circ}\right)$ & $(\mathrm{dB})$ & $\left({ }^{\circ}\right)$ & $\left(^{\circ}\right)$ & $(\mathrm{dB})$ & $\left({ }^{\circ}\right)$ & $\left({ }^{\circ}\right)$ & $(\mathrm{dB})$ & $\left({ }^{\circ}\right)$ \\
\hline 0 & 0.3 & 32 & 0 & 5.9 & 30 & 0 & 9.3 & 28 & 0.0 \\
\hline 45 & -43.8 & 32 & 0.9 & -39.9 & 30 & -0.8 & -36.1 & 28 & -0.4 \\
\hline 60 & -58.8 & 32 & 0.9 & -55.1 & 30 & -1.0 & -51.3 & 28 & -0.6 \\
\hline 90 & -89.4 & 31 & 0.3 & -85.0 & 30 & -0.9 & -80.4 & 27 & 0.3 \\
\hline 120 & -120.0 & 32 & -0.3 & -114.8 & 30 & -0.7 & -110.4 & 27 & 0.3 \\
\hline 135 & -135.3 & 32 & -0.6 & -129.5 & 30 & -0.4 & -125.6 & 27 & 0.1 \\
\hline 180 & -179.7 & 32 & 0 & -174.1 & 30 & 0 & -170.7 & 28 & 0 \\
\hline
\end{tabular}

where $\phi_{\mathrm{XPD}}$ is computed using the methods described in Sect. 2.2 and $\phi_{\mathrm{XPD}}^{n r}$ is the angle $\phi_{\mathrm{XPD}}$ for the non-rotated feed, $\phi_{\mathrm{XPD}}^{n r}=\phi_{\mathrm{XPD}}\left(\psi_{\mathrm{FP}}=0^{\circ}\right)$.

\section{Simulation results}

In what follows, we shall focus on determining this residual angle, along with the associated angle $\phi_{\mathrm{XPD}}$ and power difference $\Delta W\left(\phi_{\mathrm{XPD}}\right)$, making use of the PO simulations described in Sect. A.1. In the present study we compute the sky beam patterns for PLANCK-LFI 30 and $100 \mathrm{GHz}$ feeds at different positions in the focal plane unit, for a range of different orientations of the feed. The orientations studied correspond to rotations around the symmetry axis of the feed by angles 6 $\psi_{\mathrm{FP}}=0^{\circ}, 45^{\circ}, 60^{\circ}, 90^{\circ}, 120^{\circ}, 135^{\circ}$ and $180^{\circ}$. In particular, we carry out numerical simulations of the far-field patterns fed by corrugated horns located at three different positions in the focal plane ranging from very close to the optical axis, to the edge of the field of view (see Fig. A.3).

This will allow us to draw some general conclusions about the effect of the feed positioning on the preservation of the polarization properties through the antenna. We emphasize that,

\footnotetext{
${ }^{6}$ Note that, by symmetry, a feed rotation by $\psi_{\mathrm{FP}}$ is equivalent to a $\psi_{\mathrm{FP}}-180^{\circ}$ rotation, so we need not consider cases with $\psi_{\mathrm{FP}}>180^{\circ}$.
}

despite concentrating on the PLANCK experiment, our results can be useful for other $\mathrm{mm} / \mathrm{cm}$-wave polarimetric experiments with similar optical systems.

\subsection{Effect of feed position and orientation}

Tables 1-3 display the values of the residual angle $\left(\Delta_{\phi}\right)$ for all the simulated cases. These values were computed using Eq. (10). The corresponding values for $\phi_{\mathrm{XPD}}$ and $\Delta W_{\max }$ were determined using the three different methods described in Sect. 2.2. In each table, each $\Delta_{\phi}$-column shows the results for a different location of the feed in the focal plane (see Fig. A.3). We point out that, when using the 3rd Method, the main beam pattern is integrated over a solid angle centered at boresight (the north pole of the sphere) and down to a latitude $\theta_{0}=1.0^{\circ}$ and $0.5^{\circ}$ for the 30 and $100 \mathrm{GHz}$ feeds, respectively. These solid angles are chosen so as to include, at least, all radiated power down to the FWHM (see Sect. 2.2).

Our results show that the estimated differential rotation of the polarization plane (see Sect. 2.3) in terms of the residual angle, $\Delta_{\phi}$, depends strongly on the definition adopted for maximum cross-polarization discrimination and the associated angle, $\phi_{\mathrm{XPD}}$. In practice, we see from the calculations that the $3 \mathrm{rd}$ Method gives what one would intuitively expect from a robust 
Table 2. Same as Table 1, with $\phi_{\mathrm{XPD}}$ being determined by the 2nd Method, as described in Sect. 2.2.

Results on the Determination of $\Delta_{\phi}$ by Maximizing the Difference Between

the Co-polar Power Peak and the Cross-polar Component (2nd Method)

\begin{tabular}{|c|c|c|c|c|c|c|c|c|c|}
\hline $30 \mathrm{GHz}$ & \multicolumn{3}{|c|}{ Position 1} & \multicolumn{3}{|c|}{ Position 4} & \multicolumn{3}{|c|}{ Position 27} \\
\hline $\begin{array}{c}\psi_{\mathrm{FP}} \\
\left({ }^{\circ}\right)\end{array}$ & $\begin{array}{c}\phi_{\mathrm{XPD}} \\
\left(^{\circ}\right)\end{array}$ & $\begin{array}{c}\Delta W_{\text {Max }} \\
(\mathrm{dB})\end{array}$ & $\begin{array}{l}\Delta_{\phi} \\
\left({ }^{\circ}\right)\end{array}$ & $\begin{array}{c}\phi_{\mathrm{XPD}} \\
\left({ }^{\circ}\right)\end{array}$ & $\begin{array}{c}\Delta W_{\mathrm{Max}} \\
(\mathrm{dB})\end{array}$ & $\begin{array}{l}\Delta_{\phi} \\
\left(^{\circ}\right)\end{array}$ & $\begin{array}{c}\phi_{\mathrm{XPD}} \\
\left(^{\circ}\right)\end{array}$ & $\begin{array}{c}\Delta W_{\mathrm{Max}} \\
(\mathrm{dB})\end{array}$ & $\begin{array}{l}\Delta_{\phi} \\
\left({ }^{\circ}\right)\end{array}$ \\
\hline 0 & 0.5 & 60 & 0 & 5.7 & 56 & 0 & 8.9 & 43 & 0 \\
\hline 45 & -44.4 & 60 & 0.1 & -39.4 & 65 & -0.1 & -36.4 & 51 & -0.3 \\
\hline 60 & -59.4 & 60 & 0.1 & -54.5 & 61 & -0.2 & -51.5 & 53 & -0.4 \\
\hline 90 & -89.5 & 60 & 0 & -84.5 & 76 & -0.2 & -81.5 & 50 & -0.4 \\
\hline 120 & -119.5 & 61 & 0 & -114.4 & 57 & -0.1 & -111.4 & 44 & -0.3 \\
\hline 135 & -134.5 & 61 & 0 & -129.4 & 54 & -0.1 & -126.3 & 43 & -0.2 \\
\hline 180 & -179.5 & 60 & 0 & -174.3 & 56 & 0 & -171.1 & 43 & 0 \\
\hline $100 \mathrm{GHz}$ & & sition 1 & & & osition 4 & & & sition 2 & \\
\hline $\begin{array}{c}\psi_{\mathrm{FP}} \\
\left({ }^{\circ}\right)\end{array}$ & $\begin{array}{c}\phi_{\mathrm{XPD}} \\
\left({ }^{\circ}\right)\end{array}$ & $\begin{array}{c}\Delta W_{\mathrm{Max}} \\
(\mathrm{dB})\end{array}$ & $\begin{array}{l}\Delta_{\phi} \\
\left({ }^{\circ}\right)\end{array}$ & $\begin{array}{c}\phi_{\mathrm{XPD}} \\
\left(^{\circ}\right)\end{array}$ & $\begin{array}{c}\Delta W_{\mathrm{Max}} \\
(\mathrm{dB})\end{array}$ & $\begin{array}{l}\Delta_{\phi} \\
\left({ }^{\circ}\right)\end{array}$ & $\begin{array}{c}\phi_{\mathrm{XPD}} \\
\left({ }^{\circ}\right)\end{array}$ & $\begin{array}{c}\Delta W_{\mathrm{Max}} \\
(\mathrm{dB})\end{array}$ & $\begin{array}{l}\Delta_{\phi} \\
\left({ }^{\circ}\right)\end{array}$ \\
\hline 0 & 0.5 & 64 & 0 & 6.1 & 56 & 0 & 9.2 & 38 & 0 \\
\hline 45 & -44.4 & 58 & 0.1 & -39.5 & 61 & -0.6 & -36.8 & 38 & -1.0 \\
\hline 60 & -59.4 & 57 & 0.1 & -54.7 & 58 & -0.8 & -52.1 & 38 & -1.3 \\
\hline 90 & -89.5 & 61 & 0 & -84.8 & 59 & -0.9 & -82.2 & 37 & -1.4 \\
\hline 120 & -119.5 & 69 & 0 & -114.4 & 66 & -0.5 & -111.5 & 37 & -0.7 \\
\hline 135 & -134.5 & 62 & 0 & -129.2 & 61 & -0.3 & -126.1 & 37 & -0.3 \\
\hline 180 & -179.5 & 64 & 0 & -173.9 & 56 & 0 & -170.8 & 38 & 0 \\
\hline
\end{tabular}

Table 3. Same as Table 1, with $\phi_{\mathrm{XPD}}$ being determined by the 3rd Method, as described in Sect. 2.2.

Results on the determination of $\Delta_{\phi}$ by Maximizing the Difference

Between the Integrated Co- and Cross-polar Power Beams (3rd Method)

\begin{tabular}{|c|c|c|c|c|c|c|c|c|c|}
\hline $30 \mathrm{GHz}$ & \multicolumn{3}{|c|}{ Position 1} & \multicolumn{3}{|c|}{ Position 4} & \multicolumn{3}{|c|}{ Position 27} \\
\hline$\psi_{\mathrm{FP}}$ & $\phi_{\mathrm{XPD}}$ & $\Delta W_{\text {Max }}$ & $\Delta_{\phi}$ & $\phi_{\mathrm{XPD}}$ & $\Delta W_{\operatorname{Max}}$ & $\Delta_{\phi}$ & $\phi_{\mathrm{XPD}}$ & $\Delta W_{\text {Max }}$ & $\Delta_{\phi}$ \\
\hline$\left(^{\circ}\right)$ & $\left(^{\circ}\right)$ & $(\mathrm{dB})$ & $\left({ }^{\circ}\right)$ & $\left(^{\circ}\right)$ & $(\mathrm{dB})$ & $\left({ }^{\circ}\right)$ & $\left(^{\circ}\right)$ & $(\mathrm{dB})$ & $\left({ }^{\circ}\right)$ \\
\hline 0 & 0.5 & 32 & 0 & 5.6 & 28 & 0 & 8.7 & 25 & 0 \\
\hline 45 & -44.5 & 32 & 0 & -39.4 & 28 & 0 & -36.3 & 25 & 0 \\
\hline 60 & -59.5 & 32 & 0 & -54.4 & 28 & 0 & -51.3 & 25 & 0 \\
\hline 90 & -89.5 & 32 & 0 & -84.4 & 28 & 0 & -81.3 & 25 & 0 \\
\hline 120 & -119.5 & 32 & 0 & -114.4 & 28 & 0 & -111.3 & 25 & 0 \\
\hline 135 & -134.5 & 32 & 0 & -129.4 & 28 & 0 & -126.3 & 25 & 0 \\
\hline 180 & -179.5 & 32 & 0 & -174.4 & 28 & 0 & -171.3 & 25 & 0 \\
\hline $100 \mathrm{GHz}$ & \multicolumn{3}{|c|}{ Position 1} & \multicolumn{3}{|c|}{ Position 4} & \multicolumn{3}{|c|}{ Position 27} \\
\hline$\psi_{\mathrm{FP}}$ & $\phi_{\mathrm{XPD}}$ & $\Delta W_{\text {Max }}$ & $\Delta_{\phi}$ & $\phi_{\mathrm{XPD}}$ & $\Delta W_{\operatorname{Max}}$ & $\Delta_{\phi}$ & $\phi_{\mathrm{XPD}}$ & $\Delta W_{\text {Max }}$ & $\Delta_{\phi}$ \\
\hline$\left(^{\circ}\right)$ & $\left(^{\circ}\right)$ & $(\mathrm{dB})$ & $\left({ }^{\circ}\right)$ & $\left(^{\circ}\right)$ & $(\mathrm{dB})$ & $\left({ }^{\circ}\right)$ & $\left(^{\circ}\right)$ & $(\mathrm{dB})$ & $\left({ }^{\circ}\right)$ \\
\hline 0 & 0.5 & 33 & 0 & 5.5 & 29 & 0 & 8.7 & 26 & 0 \\
\hline 45 & -44.5 & 33 & 0 & -39.4 & 29 & 0.1 & -36.2 & 26 & 0.1 \\
\hline 60 & -59.5 & 33 & 0 & -54.4 & 29 & 0.1 & -51.2 & 26 & 0.1 \\
\hline 90 & -89.5 & 33 & 0 & -84.4 & 29 & 0.1 & -81.2 & 26 & 0.1 \\
\hline 120 & -119.5 & 33 & 0 & -114.5 & 29 & 0 & -111.3 & 26 & 0 \\
\hline 135 & -134.5 & 33 & 0 & -129.5 & 29 & 0 & -126.3 & 26 & 0 \\
\hline 180 & -179.5 & 33 & 0 & -174.5 & 29 & 0 & -171.3 & 26 & 0 \\
\hline
\end{tabular}


Table 4. Misalignment angle between the $y$-axis of both the feed and the far-field coordinate systems, for each position of the feed in the focal plane. Feed positions are shown in Fig. A.3.

\begin{tabular}{cccc}
\hline \hline Feed Position in Focal Plane & 1 & 4 & 27 \\
\hline Misalignment Angle & $0.3^{\circ}$ & $1.9^{\circ}$ & $4.3^{\circ}$ \\
\hline
\end{tabular}

estimate of cross-polarization: there is no differential rotation of the polarization plane in any of the cases studied (i.e., the residual angle, $\Delta_{\phi}$ is found to be compatible with zero in all cases, see Table 3). In fact, the maximum value of the differential rotation found $\left(\Delta_{\phi}=0.1^{\circ}\right)$ for the $100 \mathrm{GHz}$ feed at the offaxis positions 4 and 27 (see Fig. A.3), is not significant given the errors, typically of this order, coming from the accuracy in the computation of the beam pattern itself as well as its finite sampling in spherical cuts. On the other hand, Methods 1 and 2 are misleading when measuring diffuse polarization and they indicate a significant rotation of the polarization plane, whose magnitude varies systematically with frequency and position in the focal plane (see Tables 1 and 2).

In summary, we see that the 3rd Method turns out to be an appropriate definition of cross-polarization and the related cross-polar rejection angle for measurements of diffuse polarized emission. Furthermore, making use of this definition, we conclude that the PLANCK telescope does not introduce any second order (i.e. detector orientation dependent) spurious polarization.

For the absolute orientation of the polarization plane of the sky signal there is a misalignment angle introduced by the telescope optics. Referring to Figs. A.1 and A.2, by misalignment angle we mean the angle, as seen from the the $x y$-plane of the line-of-sight Cartesian coordinate system of the telescope $^{7}\left(C_{\mathrm{PM}}\right)$, between the $y$-axis of the feed coordinate system (e.g. f1) and the $y$-axis of the corresponding coordinate system of the sky beam pattern ( $\mathrm{mb} 1$ as corresponding to $\mathrm{f} 1$ ).

Table 4 shows the results for the misalignment angles, computed for each simulated position of the feed in the focal plane. They take into account the values of maximal crosspolarization rejection for the non-rotated feed, $\left(\psi_{\mathrm{FP}}^{n r}=0^{\circ}\right)$ (as determined using the 3rd Method). It can be seen that the bias or misalignment angle of the polarization plane increases as the feed moves from the center (on-axis) towards the edge of the focal plane (as we would expect), yielding an upper limit of about $5^{\circ}$. Note that these results are independent of feed orientation and frequency, within the errors (see Table 3), which means that they do not affect the differential measurement of polarization for each position of the feed. In addition, this bias can always be corrected by rearranging the orientation of each detector in the focal plane.

\subsection{Predicting far-field beam patterns}

PO modeling of a large telescope such as PLANCK requires computer intensive calculations with processing time scaling

\footnotetext{
7 By definition, the $z$-axis of the line-of sight coordinate system points to the direction of maximum power radiated (or received) by an on-axis detector.
}

as the fourth power of frequency. Moreover, modeling the response of a focal plane array including many detectors at several frequencies becomes an extremely demanding task. Therefore, we investigate to what extent it is possible to use a known (pre-computed) model of the main beam response (i.e., the peak of detector angular response), for a given feed and focal plane layout, to estimate or predict the sky main beam patterns for that detector in different focal plane configurations, i.e., for the feed in the same off-axis position but different polarization sensitivity directions. In order to do so, we must assess the similarity between the main beam patterns for different orientations of the radiating feed in the focal plane. At each frequency, we compare the beam pattern generated from two different feed orientations for which the far-field reference frame has been rotated by $\phi_{\mathrm{XPD}}$ (with its co-polar component aligned with the principal plane of polarization). Figure 1 shows the differences (in $\mathrm{dB}$ units) between the power contours of several pairs of main beam patterns. For illustrative purposes, we display the difference contours for the case where the definitions of maximum cross-polarization discrimination discussed in Sect. 2.2 disagree the most (Figs. 1a, b) and for the cases where the feeds are closest to the center of the focal plane (Figs. 1c, d).

As can be seen from Fig. 1, co-polar power differences are always below $3.0 \mathrm{~dB}$, except for a few point-like regions, while cross-polar power mismatches are locally found up to $15 \mathrm{~dB}$. Since cross-polarization peaks are typically about $30 \mathrm{~dB}$ bellow the co-polar peak (see $\Delta W_{\text {Max }}$ values in Table 3 ), it is sufficient to concentrate on the dominant (co-polar) component of the polarized beam pattern to estimate how much the beam pattern changes as a function of the feed orientation. No significant difference is found between the co-polar patterns in Figs. 1a and $b$ and therefore we conclude that these difference contours cannot be used to determine a robust definition for cross-polarization.

More interestingly, from Fig. 1, we conclude that using a pre-computed sky main beam pattern from a feed at a given frequency and orientation in the focal plane (and the corresponding maximal cross-polarization rejection angle, $\left.\phi_{\mathrm{XPD}}\right)$, it is possible to predict the shape of the main beam pattern in the sky for any other orientation of the same feed, within $\sim 3 \mathrm{~dB}$ $(\sim 15 \mathrm{~dB})$ for the the co-polar (cross-polar) pattern.

\subsection{Spurious optical polarization}

The presence of cross-polarization and the differences in the shape of the polarized beams patterns from a given feed with different orientations around its symmetry axis will result in an observation of a polarized signal not present in the sky, but rather generated by the instrument asymmetries. This is what we shall call "spurious optical polarization". 

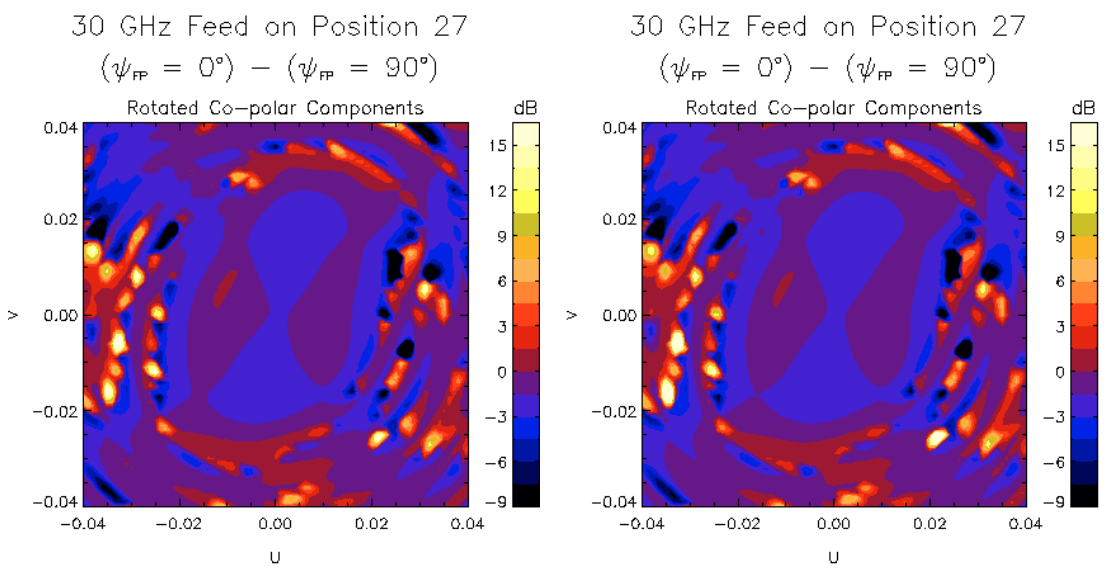

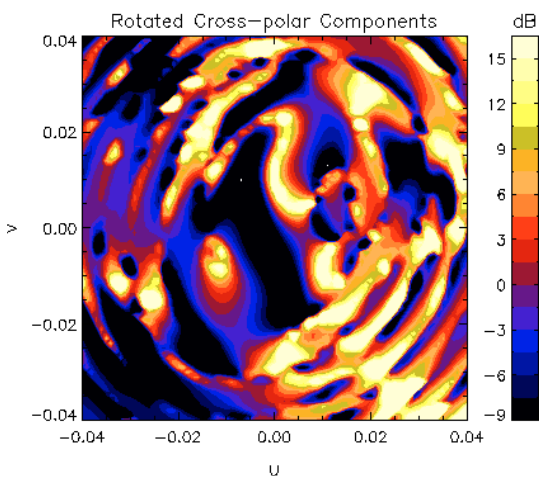

(a) 1st Method
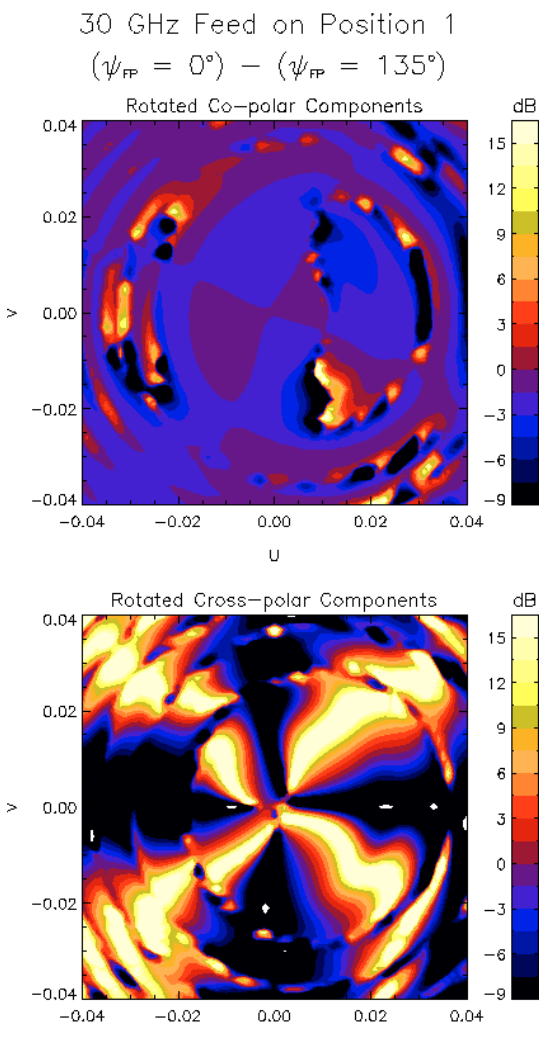

(c) 3rd Method

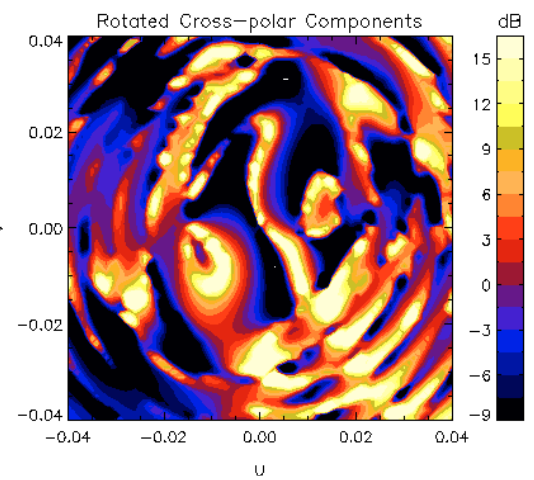

(b) 3rd Method
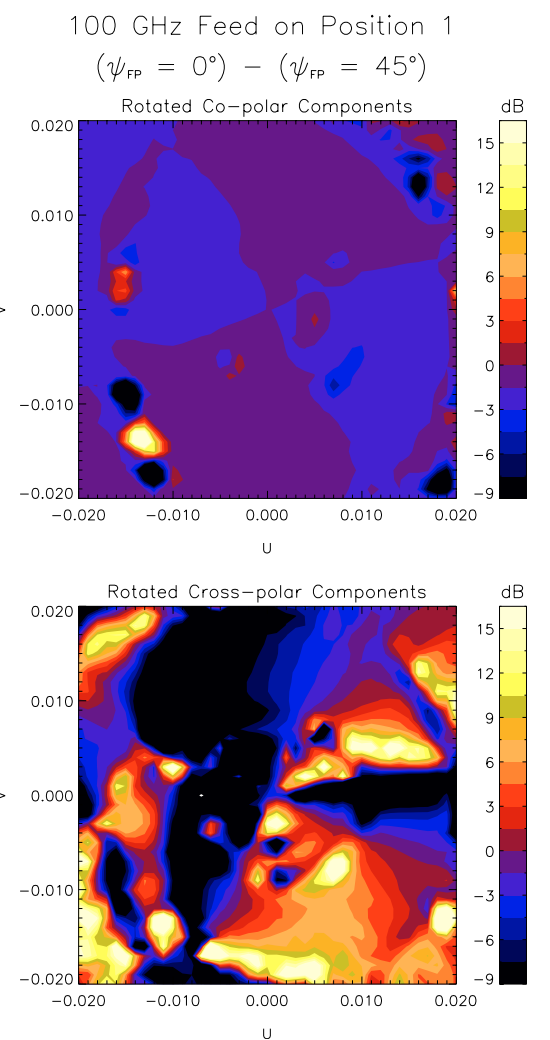

(d) 3rd Method

Fig. 1. Differences (in dB units) between main beam patterns in far field reference frames rotated by $\phi_{\mathrm{XPD}}$, for feeds at different positions and orientations in the focal plane. Cross-polarization definitions and $\phi_{\mathrm{XPD}}$ angles are determined by Methods 1 and 3 described in Sect. 2.2 (see Tables 1 and 3). 
Table 5. Normalized Stokes parameters $q_{B}$ and $u_{B}$ for different limits of integration $\theta_{0}$; at FWHM, $3^{\circ}$ and $20^{\circ}$. These parameters represent the optical spurious polarization introduced by PLANCK telescope optics for the studied focal plane configuration (in bold) and other study cases (see text).

\begin{tabular}{|c|c|c|c|c|c|c|c|c|c|}
\hline \multirow{2}{*}{$\frac{\mathbf{3 0} \mathbf{G H z}}{\theta_{0}}$} & \multicolumn{3}{|c|}{ Position 1} & \multicolumn{3}{|c|}{ Position 4} & \multicolumn{3}{|c|}{ Position 27} \\
\hline & $0.5^{\circ}$ & $3.0^{\circ}$ & $20.0^{\circ}$ & $0.5^{\circ}$ & $3.0^{\circ}$ & $20.0^{\circ}$ & $0.5^{\circ}$ & $3.0^{\circ}$ & $20.0^{\circ}$ \\
\hline$q_{B}(\%)$ & -0.0391 & -0.275 & -0.275 & 1.79 & 3.17 & 3.10 & -0.0057 & -0.131 & -0.131 \\
\hline$u_{B}(\%)$ & 0.00365 & 0.00117 & 0.00110 & 5.2 & 5.1 & 5.0 & $-\mathbf{0 . 4 2 7}$ & -0.204 & -0.205 \\
\hline $100 \mathrm{GHz}$ & \multicolumn{3}{|c|}{ Position 1} & \multicolumn{3}{|c|}{ Position 4} & \multicolumn{3}{|c|}{ Position 27} \\
\hline$\theta_{0}$ & $0.2^{\circ}$ & $3.0^{\circ}$ & $20.0^{\circ}$ & $0.2^{\circ}$ & $3.0^{\circ}$ & $20.0^{\circ}$ & $0.2^{\circ}$ & $3.0^{\circ}$ & $20.0^{\circ}$ \\
\hline$q_{B}(\%)$ & 0.164 & 0.093 & 0.093 & -0.138 & -0.0398 & -0.0422 & 1.42 & 2.05 & 2.05 \\
\hline$u_{B}(\%)$ & -0.00418 & 0.0052 & 0.0052 & -0.51 & -0.086 & -0.086 & 2.22 & 3.44 & 3.43 \\
\hline
\end{tabular}

We quantify this effect in the modeled telescope as the response of the system to a diffuse unpolarized sky signal, in terms of normalized Stokes parameters. Following standard conventions (Kraus 1982), PLANCK will measure Stokes parameters $I, Q$ and $U$ by combining the output of four linearly polarized detectors; a first pair having principal planes of polarization at right angles to each other and a second pair with principal planes of polarization at right angles as well, but rotated by $45^{\circ}$ with respect to the first pair of detectors. For this purpose we shall use the simulations results for the 30 and $100 \mathrm{GHz}$ feeds at different positions in the focal plane unit and orientations $\phi_{\mathrm{FP}}=0^{\circ}, 45^{\circ}, 90^{\circ}, 135^{\circ}$.

The power available to the detectors is proportional to the convolution of the beam with the sky signal, i.e.

$P \propto \int_{4 \pi} \mathrm{d} \Omega\left(\boldsymbol{S}_{B} \cdot \boldsymbol{S}_{S}\right)$

where $S_{B}=\left(I_{B}, Q_{B}, U_{B}, V_{B}\right)$ is the Stokes vector of the beam on the sky and $\boldsymbol{S}_{S}$ is that of the sky signal. If the sky signal is uniform, then

$P \propto \int_{4 \pi} \mathrm{d} \Omega\left(E_{\mathrm{co}}^{2}+E_{\mathrm{cr}}^{2}\right)$

We shall use normalized Stokes parameters, $q_{B}=Q_{B} / I_{B}$, and $u_{B}=U_{B} / I_{B}$, to characterize the spurious optical polarization,

$q_{B}=\frac{P\left(\psi_{1}\right)-P\left(\psi_{1}+\frac{\pi}{2}\right)}{P\left(\psi_{1}\right)+P\left(\psi_{1}+\frac{\pi}{2}\right)} ; \quad u_{B}=\frac{P\left(\psi_{2}\right)-P\left(\psi_{2}+\frac{\pi}{2}\right)}{P\left(\psi_{1}\right)+P\left(\psi_{1}+\frac{\pi}{2}\right)}$

where $P\left(\psi_{1}\right)+P\left(\psi_{1}+\frac{\pi}{2}\right) \equiv P\left(\psi_{2}\right)+P\left(\psi_{2}+\frac{\pi}{2}\right)$ and $P(\psi)$ is the total power integrated over the beam (within a co-latitude range $\left.\left[-\theta_{0} ;+\theta_{0}\right]\right) \cdot \psi_{2}-\psi_{1}=45^{\circ}$ is the relative phase shift in the polarization planes. The calculated estimates of those parameters for the modeled feeds are shown in Table 5 for different limits of integration $\theta_{0}$. It can be seen that $q_{B}$ and $u_{B}$ depend on the chosen integration limit, if it less than a few times the FWHM.

Table 5 also shows that the spurious polarization depends strongly on location and frequency and that a high level of up to $5 \%$ polarized contamination of the diffuse unpolarized intensity is found for feeds at 30 and $100 \mathrm{GHz}$. The observed large variation of this level across the focal plane seems, at first glance, difficult to explain. In order to illustrate the issue, we show in Fig. 2 the dependence of $q_{B}$ and $u_{B}$ across the main beam for the two worst cases in Table 5 ; the $30 \mathrm{GHz}$ feed on position 4 (Fig. 2a) and the $100 \mathrm{GHz}$ feed on position 27 (Fig. 2c) and for the cases where the two feeds are in their actual positions in the studied PLANCK focal plane layout; the $30 \mathrm{GHz}$ feed on position 27 (Fig. 2b) and the $100 \mathrm{GHz}$ feed on position 4 (Fig. 2d).

It can be seen that $q_{B}$ and $u_{B}$ exhibit large fluctuations across the main beam, which may cancel out more effectively when integrated in angular space. The final integrated values of $q_{B}$ and $u_{B}$ will therefore depend on the fine details of the modeled patterns, which are affected by the exact location of the feed in the focal plane. We can expect that, in a real system where the detectors are sensitive to wide bandwidths, part of the fluctuations will be averaged out. In addition, it is not clear to what extent the fine details of the models will be reproduced by the real physical systems. For these reasons we can consider that the levels of spurious optical polarization here estimated are very conservative upper limits.

It is however emphasized that, given the expected small relative amplitude of the polarized with respect to the unpolarized CMB signal (about 10\%), this upper limit of opticallyinduced polarization cannot be ignored. At these frequencies, there are two large scale sources of unpolarized diffuse components in the sky: the isotropic $\mathrm{CMB}$ emission (at $T \simeq 2.73 \mathrm{~K}$ ) and the $\mathrm{CMB}$ dipole, a Doppler temperature anisotropy in the sky of amplitude $\sim 3 \mathrm{mK}$ (see, e.g. Lineweaver et al. 1996). As PLANCK scans the sky, spurious optical polarization will introduce a constant bias for the former and a slowly varying bias for the latter. The level of these biases $(\sim 30 \mathrm{mK}$ and $0.03 \mathrm{mK}$, for each $1 \%$ of spurious polarization) is quite large compared to the expected $\mathrm{CMB}$ polarized signal of $\sim 5-10 \mu \mathrm{K}$. Note however that a constant bias does not affect the measurements of small scale anisotropies, and a slowly varying bias is likely to be easily removable using calibration procedures.

The main source of concern in the detection of polarized sky signals for a PLANCK-like experiment will arise from the spurious signal that leaks from the measured unpolarized intensity itself. The percentage of spurious polarization is, in the 


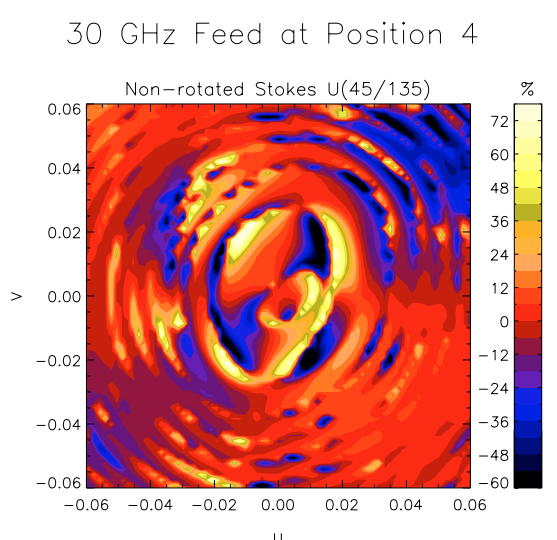

(a)

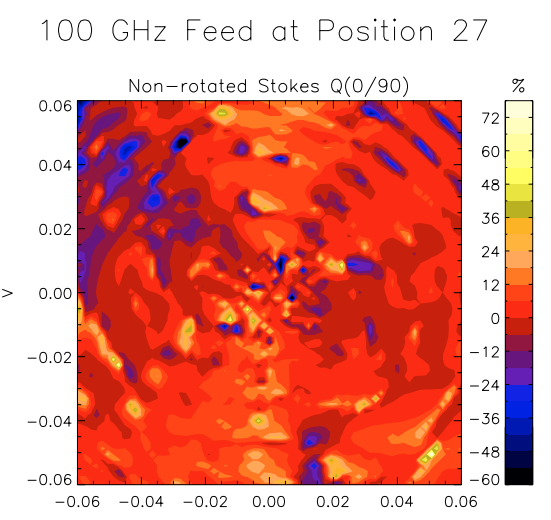

(c)

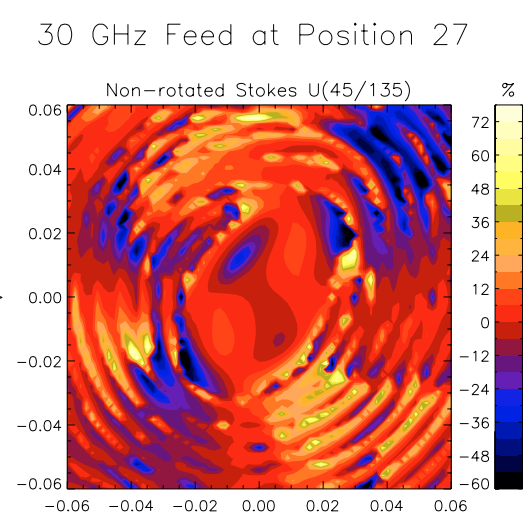

(b)

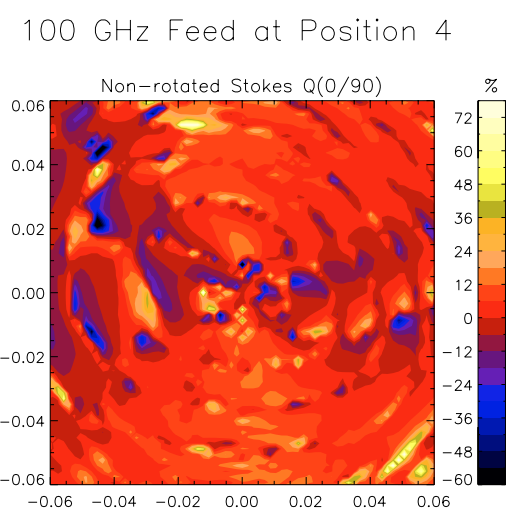

(d)

Fig. 2. Contour plot of the distribution of the normalized Stokes parameters across the main beam (with $|\theta|<3^{\circ}$ ). The plot shows one of the parameters $\left(u_{B}\right.$ or $q_{B}$ ) for the two worst study cases in Table 5; a) the $30 \mathrm{GHz}$ feed on position 4 and c) the $100 \mathrm{GHz}$ feed on position 27. The same parameters are also shown for the feeds in their real positions in the studied PLANCK focal plane layout: b) the $30 \mathrm{GHz}$ feed on position 27 and d) the $100 \mathrm{GHz}$ feed on position 4.

very worst cases, only factors of a few smaller than the percentage of polarization signal. Potentially this spurious level will ultimately introduce a fundamental limit on the measurement of CMB polarization anisotropies at sub-degree scales, i.e. at angular scales comparable to the main-beam size. It is however emphasized that the spurious polarization estimated for the actual positions of the PLANCK feeds is much lower than in the worst case scenario, and it is certainly below the expected polarized CMB signal. Moreover, this situation should be further improved if we take into account other relevant effects such as the large detector bandwidth that tends to suppress to a large extent the small-scale angular variations observed on monochromatic radiation patterns.

\subsection{Model uncertainties}

This study has not taken into account imperfections in the optics due to the effects of dust contamination, microcracks, asymmetry of the focal plane, or the effects of optical misalignment, since it is today very difficult to model such effects. However, qualitatively it can be argued that:

- As long as dust grains are not elongated and preferentially aligned, their effect is largely to scatter power from the main beam into the far sidelobes. In this study we are interested in the polarization introduced through the main beam and therefore dust can be safely ignored.

- The effect of microcracks is qualitatively similar to that of dust with the exception that elongation and preferential alignment are expected. Fortunately, in the case of PLANCK, all technological indications are that no microcracks will be present.

- Misalignment introduces additional aberration in the optics that translates into distortion of the main beam. Distortion is always related to depolarisation and therefore it is of potential concern. However, the aberrations introduced by misalignment are, by design and test, kept at a level which is a small fraction of the intrinsic optical distortions. Therefore it is reasonable in this initial study to assume that it will remain a second order effect.

- An asymmetric focal plane will likely introduce differential effects in the response of a single horn to different planes of polarization. However, the effects of mutual coupling between PLANCK horns that are adjacent in the focal plane are kept, by design, at a rather low level with respect to the main beam (i.e, at least $30 \mathrm{~dB}$ below peak) and we assume here that they are not affecting the main results of this study. 
In PLANCK, the higher frequency detector chains include also a number of optical elements (such as filters, detector embedding structures, cavities, etc.) which potentially introduce polarization effects. Such chains have been measured and found to introduce very low levels of cross-polarization. However, since they are of the same order as the effects listed above, they should be included in future simulations.

Finally, we emphasize that this study is based on a numerical model, which naturally is subject to some degree of uncertainty when compared to reality. In this respect, the software used for our simulations (GRASP) is today considered to be the benchmark software tool for analysis of large antenna systems. Recent studies (Murphy et al. 2001) have been carried out to investigate the reliability of a variety of software tools, including GRASP8, in modelling optical systems in the submillimetre wave band. In these studies, simulated results from different software packages were compared to each other as well as to experimental measurements. These studies show GRASP to be the most reliable package, agreeing with experimental measurements down to a $-40 \mathrm{~dB}$ level in predicting the amplitude pattern and showing the best agreement in phase measurements. Moreover, the same study has shown that GRASP8 is the only available package capable of handling polarization in the case of off-axis reflectors systems.

Therefore, in terms of model uncertainties, it is considered that this study is as close to real as can be expected, within the assumptions made. However, it is acknowledged that the real system performance must ultimately be measured. A comprehensive pre-launch test campaign is planned for PLANCK; these measurements will be later combined with in-flight characterisation to obtain the final system performance.

\section{Summary and conclusions}

We have studied the systematic effects introduced by the PLANCK optical system in the measurement of polarized signals. For the purpose of this study we have carried out PO simulations of the former PLANCK telescope configuration with the 30 and $100 \mathrm{GHz}$ feeds at three different feed locations (see Fig. A.3) and several orientations. From the analysis of these simulations, our results (see Sect. 3) yield the following main conclusions:

- The relative orientation of polarization planes of diffuse sky signals is near-perfectly preserved by the optics, so that no systematic effects in the measurement of polarization are to be expected from the feed orientations in the current baseline. This symmetry is only observed when a proper definition of cross-polarization for non-ideal antennas is used (see Sect. 2.2).

- The absolute orientation of polarization planes is systematically rotated by the telescope. Such a misalignment angle can be as large as $5^{\circ}$ and depends only on frequency, increasing monotonically as the feed is moved towards more off-axis positions (see Table 4). However, this misalignment can be corrected by appropriate design of the focal plane.
- Due to the high symmetry of the telescope, it is possible to use a pre-computed polarization main-beam pattern for a given feed to predict main beam patterns (within $2^{\circ}$ in colatitude) for arbitrary orientations of that feed within $\sim 3 \mathrm{~dB}$ $(\sim 15 \mathrm{~dB})$ for the the co-polar (cross-polar) pattern (see Sect. 3.2). This will allow systematic studies of the polarization measurements, involving a larger number of feeds, by using a minimal amount of CPU time.

- Spurious optical polarization (i.e., polarization solely introduced by the telescope optics) is estimated to be less than $0.2 \%$ for the actual locations of the 30 and $100 \mathrm{GHz}$ feeds in the studied focal plane of PLANCK (see Fig. A.3). Other (less optimal) studied cases show spurious optical polarization as large as $\sim 5 \%$ of the unpolarized sky signal (see Table 5). It is noted however that these estimates rely on uncertain fine details in the modeled patterns and therefore should be considered as order of magnitude only. It is likely that real experiment features such as broad bands, detector responsivity and optical imperfections would affect the results, reducing the magnitude of this effect. Hence these simulations should only be used as upper limit guidelines.

Further studies on the impact of PLANCK optics on the detection of polarization should consider the following topics:

- Use of the final optical configuration of the telescope, in particular the focal plane, has recently undergone some modifications.

- Take into account second order effects (already discussed in Sect. 3.4) that may be important to the detection of polarization in the main beam region.

- Extending the studied region further into the sidelobes, to include any second order effects that may have an impact on the detection of polarization in this regions.

Finally the results of these studies will be compared to measurements obtained on qualification models of the PLANCK optics during a pre-launch test campaign, planned to start in 2004.

Acknowledgements. We wish to thank F. Villa, M. Bersanelli, R. Mandolesi for significant comments on the manuscript; the referee, J. Delabrouille for useful comments on the manuscript; G. Giardino, and A. Martín Polegre for substantial help with the GRASP8 modelling. PF acknowledges a post-doctoral CMBNet fellowship from the European Commission. GF acknowledges a scholarship from the Fundação para a Ciência e Tecnologia (FCT).

\section{Appendix A: GRASP8 simulations}

\section{A.1. Simulations setup and inputs}

As stated before, the telescope configuration used for the present analysis is an early version of the current PLANCK telescope design. It corresponds to a dual reflector optical system with an offset aplanatic geometry. The exact telescope configuration is shown in Table A.1.

Figures A.1 and A.2 show two different views of the PLANCK Telescope system, with the $100 \mathrm{GHz}$ feed located 
Table A.1. Early PLANCK Telescope configuration used for the present study (current telescope dimensions are slightly different).

\begin{tabular}{lc}
\multicolumn{1}{c}{ Main Reflector } & \\
\hline \hline Surface Shape & Off-axis Ellipsoid \\
\hline Ellipsoid Major Diameter (2a) & $22054.938 \mathrm{~mm}$ \\
\hline Inter Foci Distance (2c) & $20564.583 \mathrm{~mm}$ \\
\hline Rim Center Offset & $1038.85 \mathrm{~mm}$ \\
\hline Projected Rim Shape & Circular \\
\hline Rim Projected Aperture & $750.00 \mathrm{~mm}$ \\
\hline
\end{tabular}

\section{Sub Reflector}

\begin{tabular}{lc}
\hline \hline Surface Shape & Off-axis Ellipsoid \\
\hline Ellipsoid Major Diameter (2a) & $1641.58 \mathrm{~mm}$ \\
\hline Inter Foci Distance (2c) & $761.92 \mathrm{~mm}$ \\
\hline Rim Center Offset & $328.15 \mathrm{~mm}$ \\
\hline Projected Rim Shape & Elliptical \\
\hline Rim Major Axis Projected Aperture & $509.30 \mathrm{~mm}$ \\
\hline Rim Minor Axis Projected Aperture & $390.30 \mathrm{~mm}$ \\
\hline
\end{tabular}

in three different positions in the focal plane (positions 1, 4 and 27, also shown with more detail in Fig. A.3).

Reference frames f1, f4 and f27 are used to describe the three different positions of the feed in the focal plane while reference frames mb1, mb4 and mb27 are used to describe the sky beam patterns, the latter having their $z$-axis (in blue) always pointing to the center of the main beam in the sky, set to be the maximum of the co-polar pattern. Coordinate systems f1, $\mathrm{f} 4$ and $\mathrm{f} 27$ are defined on the basis of the coordinate system located on the vertex of the ellipse that subtends the primary mirror $\left(V_{\mathrm{PM}}\right)$ while $\mathrm{mb} 1, \mathrm{mb} 4$ and $\mathrm{mb} 27$, are defined on the basis of the coordinate system located on the center of the primary mirror $\left(C_{\mathrm{PM}}\right)$ which has the same orientation as $V_{\mathrm{PM}}$ (see Figs. A.1 and A.2). Furthermore, the orientation of $V_{\mathrm{PM}}$ is the same as the spacecraft coordinate system rotated by $+13.75^{\circ}$ around its $y$-axis.

For all simulations in this study, we used the polar coordinates $(\theta, \phi)$ that can range to cover the whole sky (i.e., $-\pi \leq$

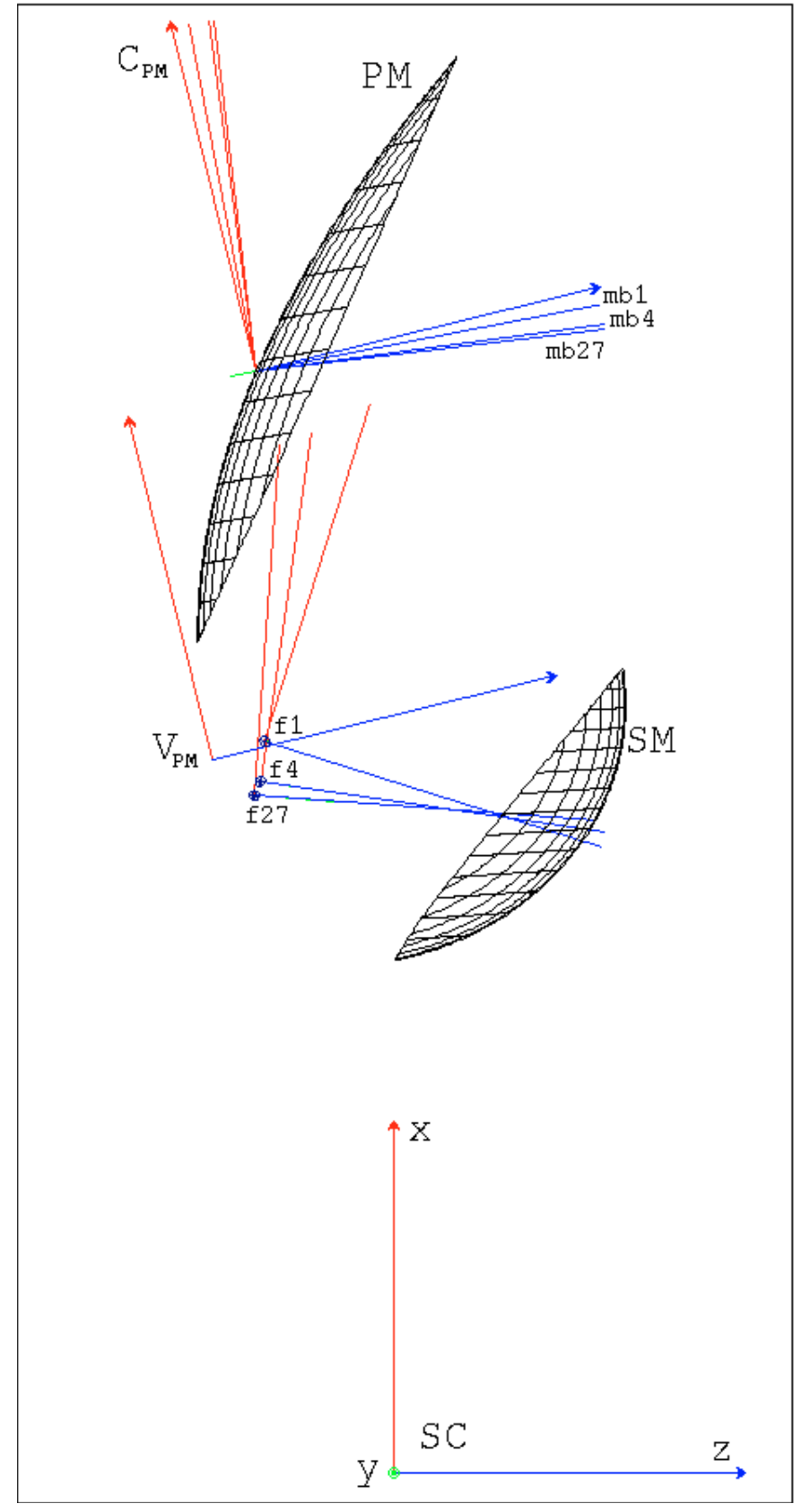

Fig. A.1. Cross-section of the objects that define the Alcatel version of PLANCK telescope in the GRASP8 model of our study. Besides the primary (PM) and secondary (SM) mirrors, this plot includes the $100 \mathrm{GHz}$ feed in three different positions in the focal plane with the corresponding coordinate systems for the feed positions (f1, f4, f27) and the different orientations of the main beam in the sky (mb1, mb4, mb27). It also includes the fixed coordinate systems for the vertex $\left(V_{\mathrm{PM}}\right)$ and the center $\left(C_{\mathrm{PM}}\right)$ of the primary mirror and for the spacecraft (SC).

$\theta \leq \pi$ and $0 \leq \phi<\pi)^{8}$ producing ASCII data files that have a structure such as to hold the values of the two complex amplitudes (real and imaginary) of the two components of the beam pattern (co- and cross-polar), for all given sets of points $(\theta, \phi)$. All simulations were done in order to determine the radiation

\footnotetext{
${ }^{8}$ Note that $\theta$ is a latitude coordinate, while $\phi$ is a longitude coordinate.
} 


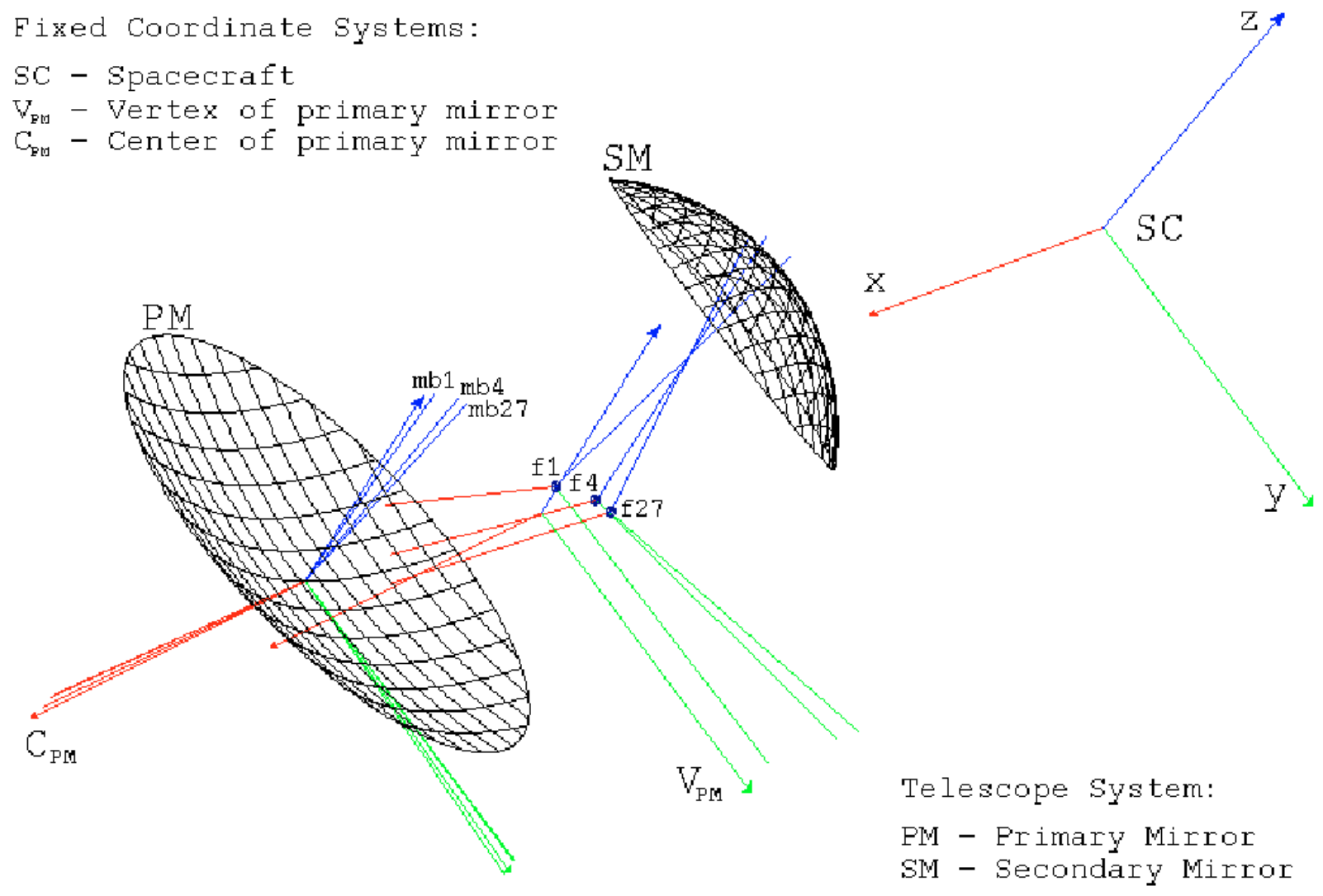

Fig. A.2. Oblique view of the objects that define the Alcatel version of PLANCK telescope in the GRASP8 input file of this study, as shown in the previous Fig. A.1.

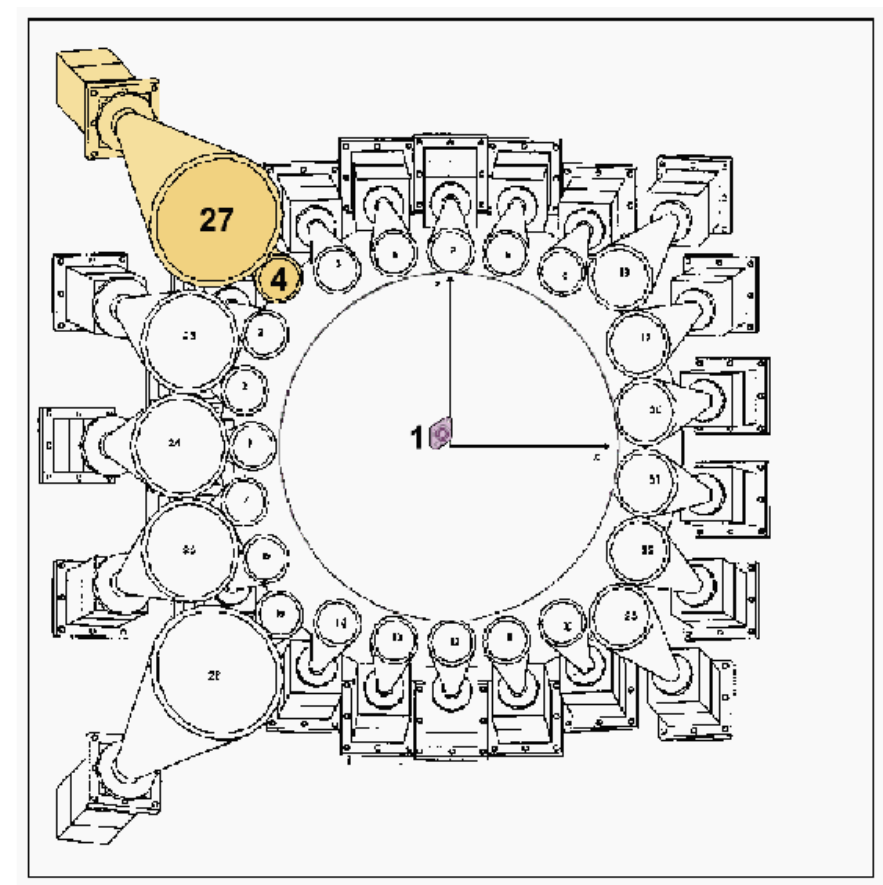

Fig. A.3. Former design of PLANCK Telescope LFI focal plane showing the detector positions used for this study: on the top left-hand corner, positions 27 and 4 with the 30 and $100 \mathrm{GHz}$ detectors, respectively and position 1 , closer to the center of the focal plane unit, with the HFI $857 \mathrm{GHz}$ detector. pattern in the sky area around the main beam. To sample this part of the sky, coordinate $\theta$ was chosen to range within the limits $-20^{\circ} \leq \theta \leq+20^{\circ}$ in a set of 401 points with intervals of $0.1^{\circ}$ while coordinate $\phi$ ranged within $0^{\circ} \leq \phi<180^{\circ}$ in a set of 36 polar cuts separated by $5^{\circ}$.

In a GRASP8 simulation, the scattered field can be calculated by using Physical Optics (PO) combined with the Physical Theory of Diffraction (PTD) or, alternatively, using Geometrical Optics (GO) combined with the Geometrical Theory of Diffraction (GTD). According to TICRA (1997), for focused reflector systems PO/PTD is intended to be used in the far field around beam maxima, whereas GTD can be used in the side-lobe region. For these reasons and since we were only interested in the main beam sky area, our simulations were done with PO/PTD calculations. In what concerns the density of the PO integration grid, to determine the number of points for which the PO currents are calculated in each reflecting surface, we referred to a formula given by TICRA (1997) where the wavelength, the reflector diameter and the maximum latitude, $\theta_{\max }$, are input parameters in the determination of the minimum number of points for which the PO integration converges.

To simulate the conical corrugated horns used in the PLANCK telescope, the input radiation signal of the feeds used in our simulations had the form of a spherical wave expansion. Figures A.5 and A.4 show the far field radiation pattern when 

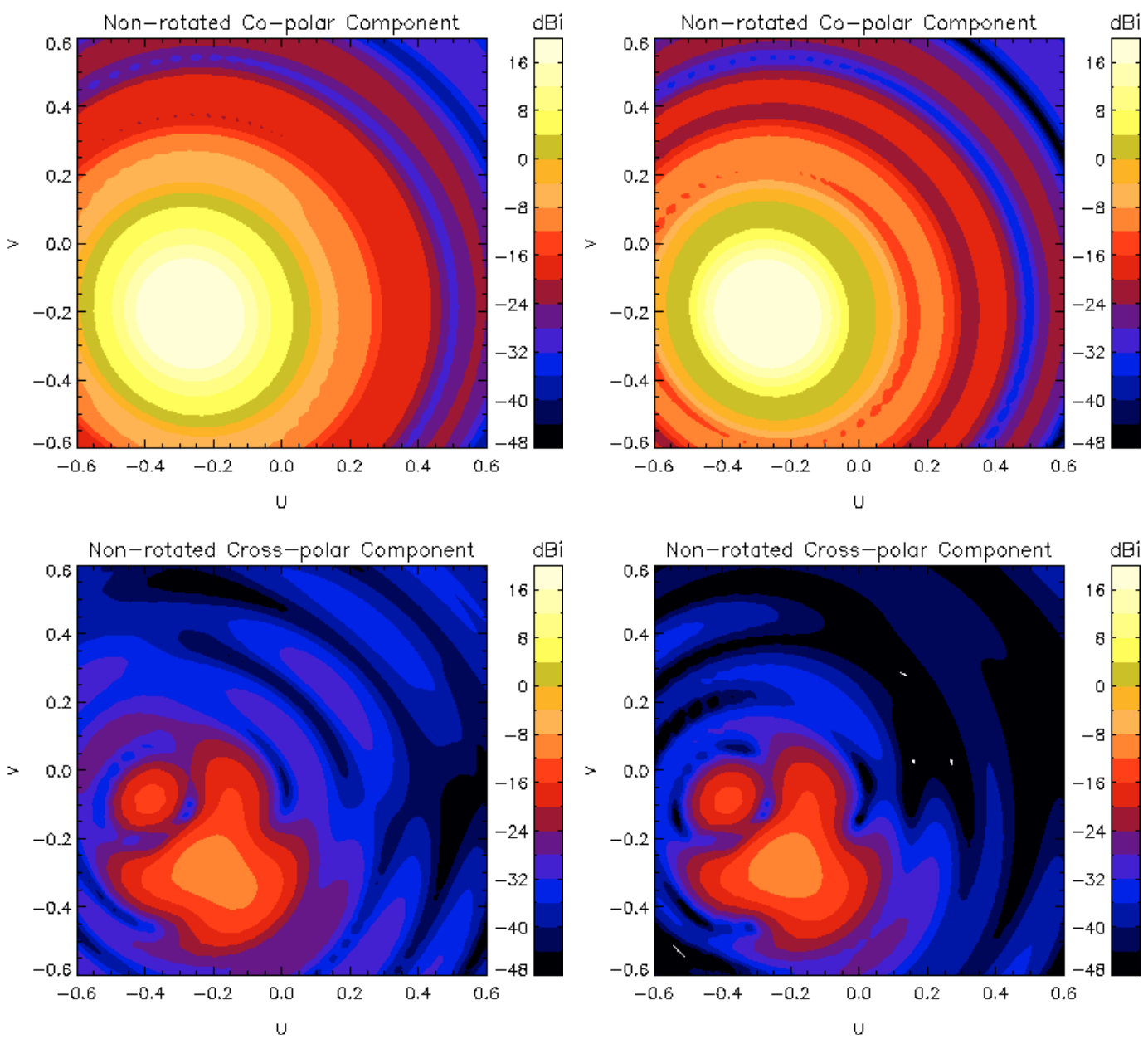

Fig. A.4. Contour plots of the far field radiation pattern $\left(|\theta|<30^{\circ}\right)$ when the feeds radiate directly to the sky, i.e., the input polarized signal does not go through the telescope system.

the input signal radiates directly from the feed to the sky, without going through the telescope system; Fig. A.5 shows the plot of the polar cut at $\phi=20^{\circ}$ while Fig. A.4 shows the whole contour in the $(U, V)$ plane.

Note that to project the polar coordinate system $(\theta, \phi)$ onto the $(U, V)$ plane we have the following transformation,

$$
\begin{array}{ll}
U=\sin \theta \cos \phi ; & -\pi \leq \theta \leq \pi \\
V=\sin \theta \sin \phi ; & 0 \leq \phi<\pi .
\end{array}
$$

In Figs. A.4 and A.5, note that the beam maxima, the co-polar peaks, are not centered at $(U, V)=(0,0)$ nor at latitude $\theta=0^{\circ}$. This off-center positioning of the beam pattern in the coordinate system $C_{\mathrm{PM}}$ when the feeds radiate directly to the sky is due to the off-axis positioning of the feeds in this same reference frame. When the radiation pattern goes through the telescope system instead of going directly from the feed to the sky, a much smaller off-center positioning of the beam can be observed in the coordinate system $C_{\mathrm{PM}}$. However, in order to have a centered beam maximum in all plots and contours, for each of the three different feed positions in the focal plane (1, 4 and 27), the coordinate system $C_{\mathrm{PM}}$ was reoriented in order to always have the $z$-axis pointing to the co-polarization power peak. This procedure originated the three different coordinate systems (mb1, mb4 and mb27), already mentioned before ${ }^{9}$.

\section{A.2. Simulations outputs and results}

Figure A.6 shows the far field radiation patterns when the input polarized signal goes through the telescope system. It can be seen that, as a result of the far field reference frame reorientation mentioned in the previous section, the co-polar peaks are always centered at $\theta=0^{\circ}$ whether the feed is on position 27 (left plot) or on position 4 (right plot). We show the contour plots of the far field radiation pattern around the main beam for various simulations. In particular, Figs. A.7 and A.8 show radiation patterns in the non-rotated far field reference frame and in the reference frame rotated by the $\phi_{\mathrm{XPD}}$ angle. This angle is determined by each one of the three different methods

\footnotetext{
9 These far field reference frames ( $\mathrm{mb} 1, \mathrm{mb} 4 \mathrm{and} \mathrm{mb} 27)$ can be seen in Figs. A.1 and A.2. Note that they have a common origin with $C_{\mathrm{PM}}$, at the center of the primary mirror, but different orientations.
} 


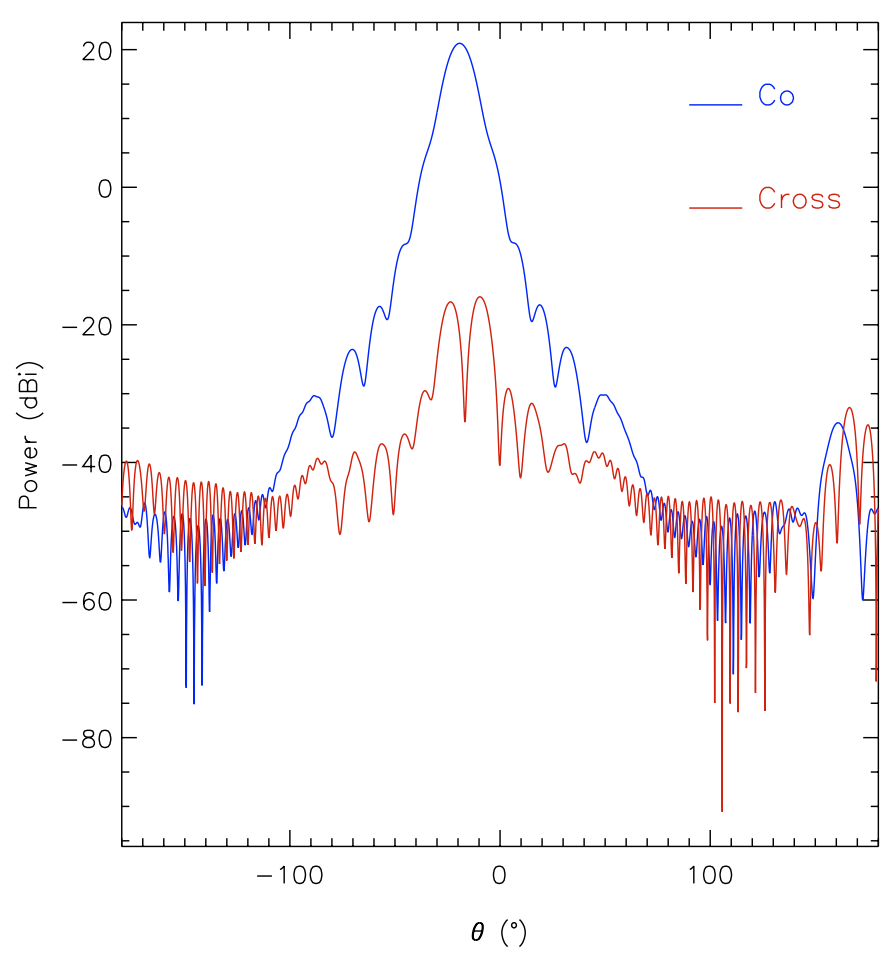

$100 \mathrm{GHz}$ Feed Radiation Pattern $\left(\phi=20^{\circ}\right)$

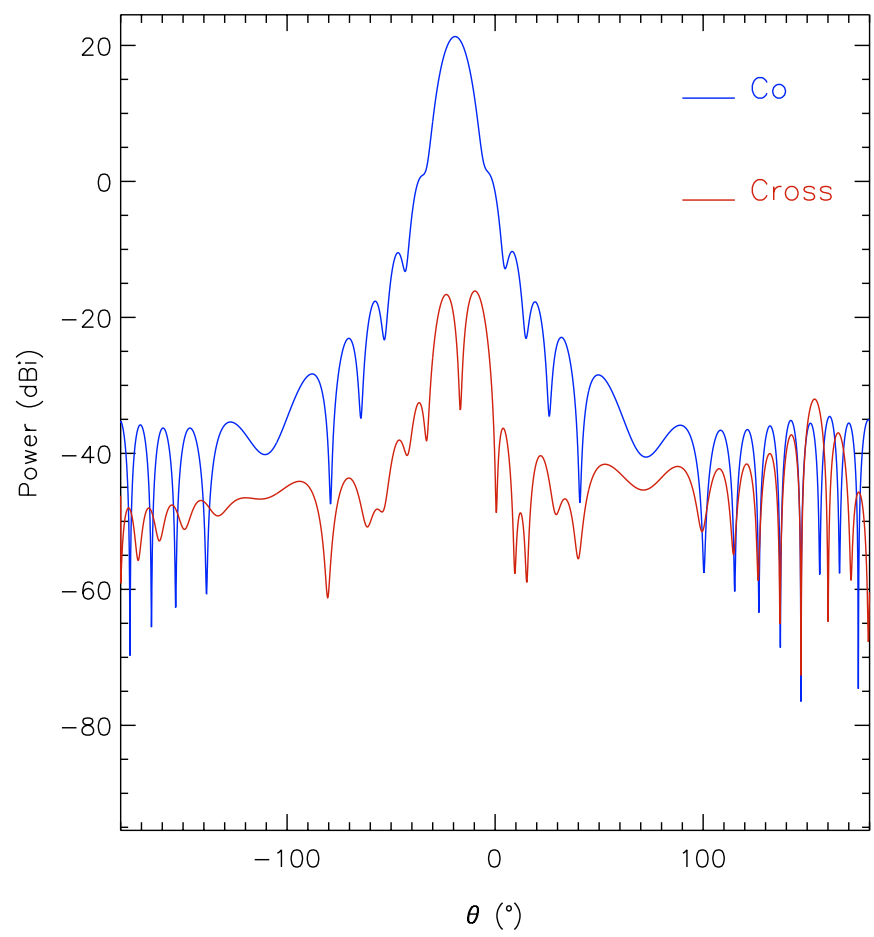

Fig. A.5. Polar cuts, at $\phi=20^{\circ}$, of the contour plots in Fig. A.4.

described in Sect. 2.1. Plots are shown side by side for an easier comparison.

As expected, for simulations with a non-rotated feed, i.e., $\psi_{\mathrm{FP}}=0^{\circ}$ (Fig. A.8), the highest peak is at the co-polar direction

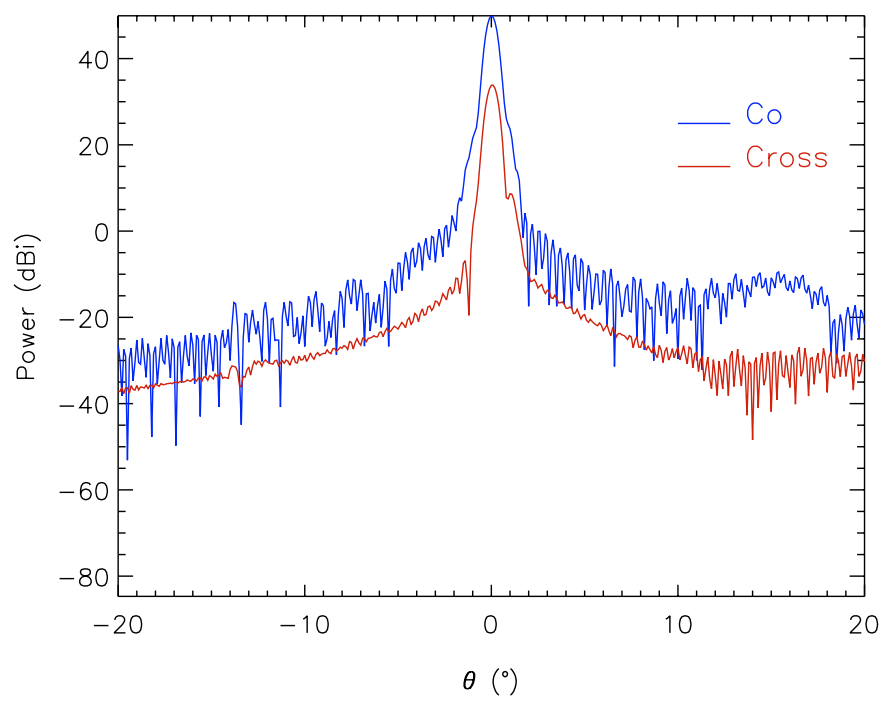

$100 \mathrm{GHz}$ Feed on Position $4\left(\phi=0^{\circ}\right)$

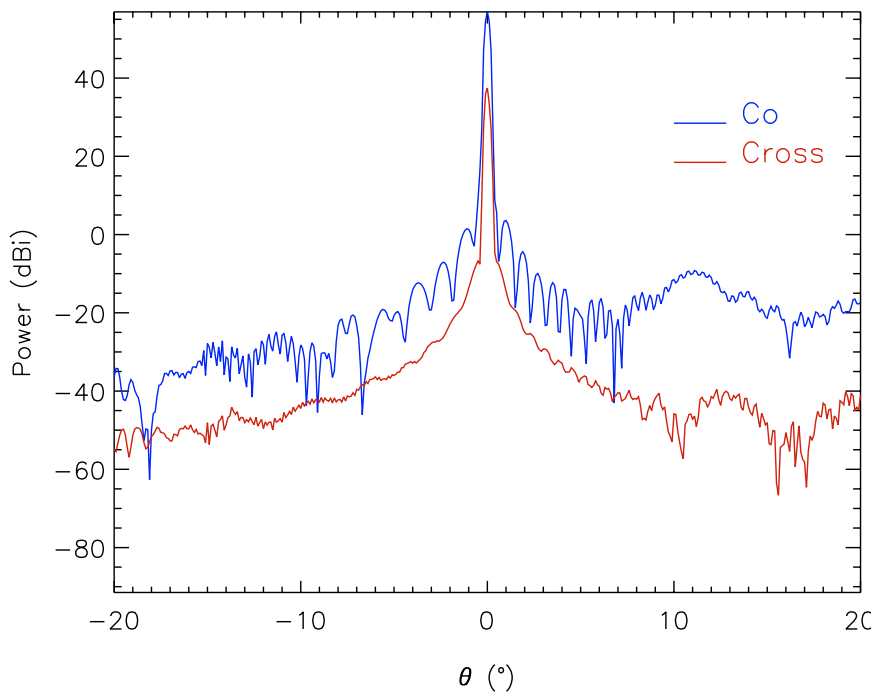

Fig. A.6. Polar cuts at $\phi=0^{\circ}$ of the 30 and $100 \mathrm{GHz}$ sky radiation patterns when the input polarized signal goes through the telescope system.

before and after the far field reference frame is rotated by $\phi_{\mathrm{XPD}}^{n r}$ (being $\phi_{X P D}^{n r}$ always a small angle), while for simulations with a feed orientation of $\psi_{\mathrm{FP}}=90^{\circ}$ (Fig. A.7), the highest peak is at the cross-polar direction before the far field reference frame is rotated by $\phi_{\text {XPD }}$ which then aligns the highest peak with the copolar direction. On the other hand and also as expected, for simulations with a feed orientation of $\psi_{\mathrm{FP}}=45^{\circ}, 135^{\circ}$ (Fig. A.9), the co- and cross-polar patterns have similar peaks before the rotation of the far field reference frame by $\phi_{\mathrm{XPD}}$. 

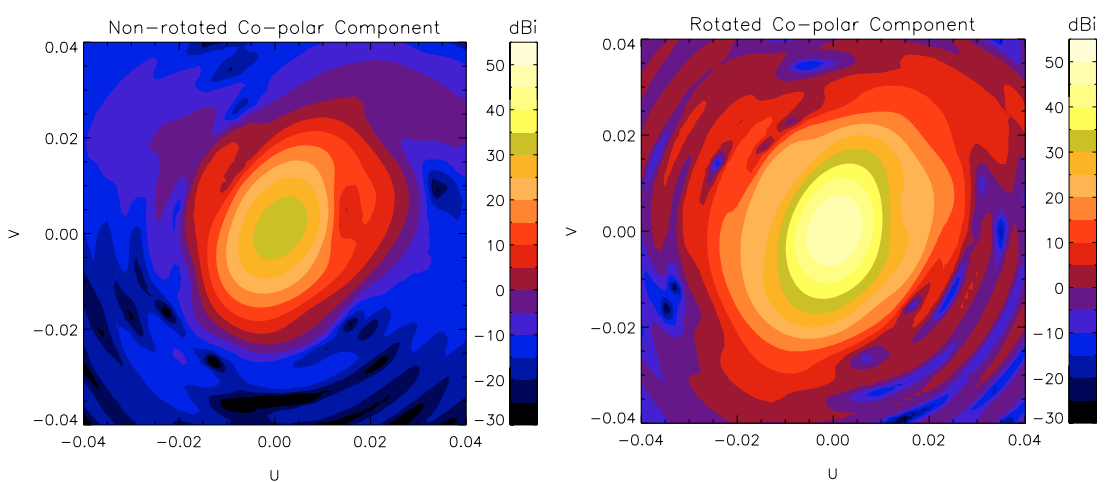

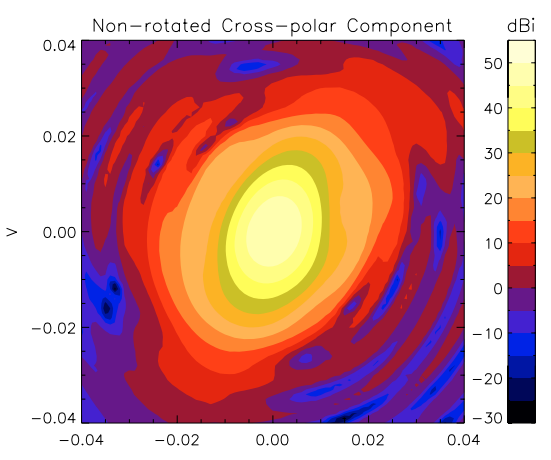

(a)

$$
\psi_{\mathrm{FP}}=90^{\circ}
$$
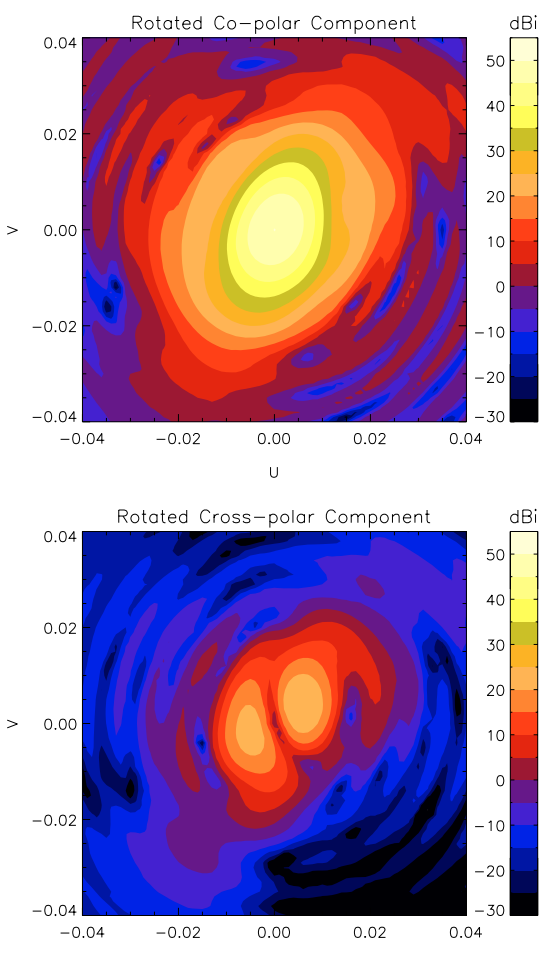

(c)

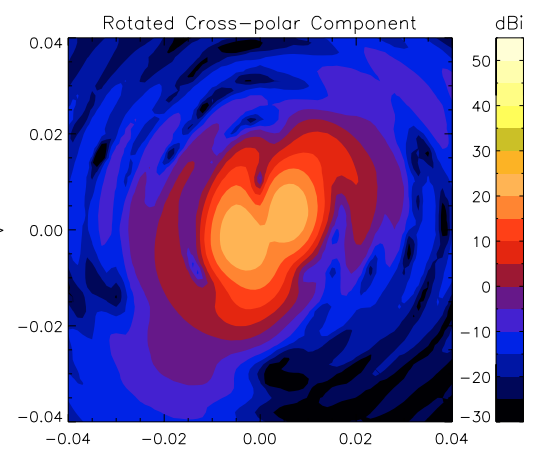

(b)

$$
\psi_{\mathrm{FP}}=90^{\circ}
$$
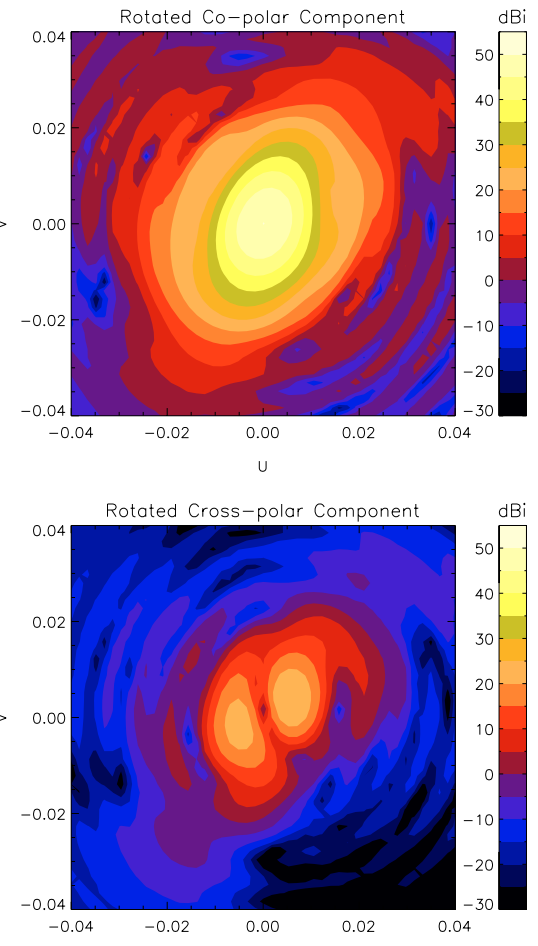

(d)

Fig. A.7. Contour plots of the Co- and Cross-polar patterns around the main beam $\left(|\theta|<2^{\circ}\right)$ for the $30 \mathrm{GHz}$ feed rotated by $\psi_{\mathrm{FP}}=90^{\circ}$ at position 27. The figure shows a) the beam pattern in the non-rotated far field reference frame and the same beam pattern in the far field reference frame rotated by b) $\phi_{\mathrm{XPD}}=-79.6^{\circ}$ (see Table 1), c) $\phi_{\mathrm{XPD}}=-81.5^{\circ}$ (see Table 2) and d) $\phi_{\mathrm{XPD}}=-81.3^{\circ}$ (see Table 3). 

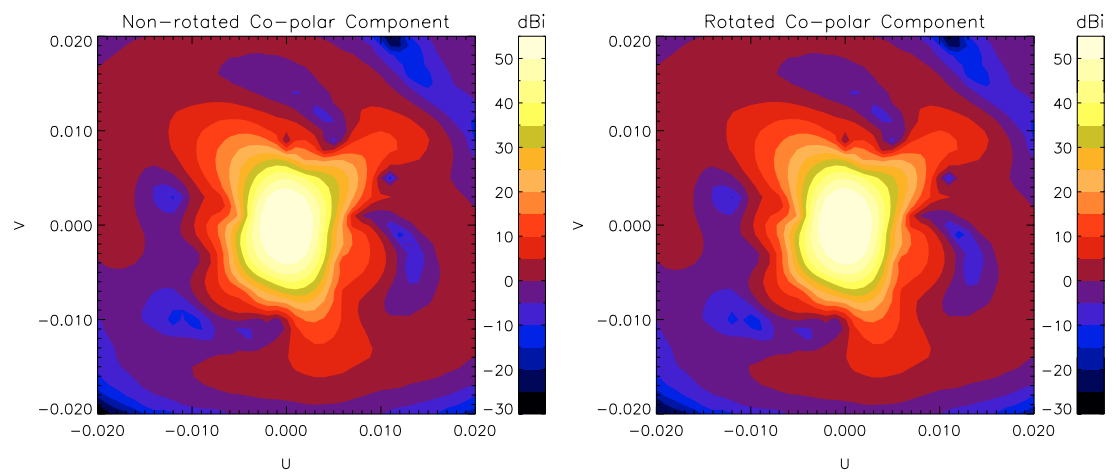

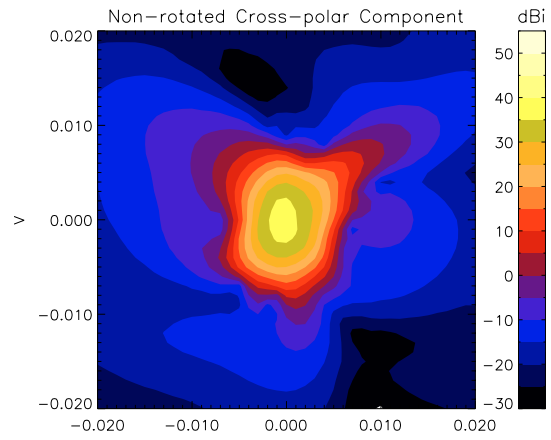

(a)
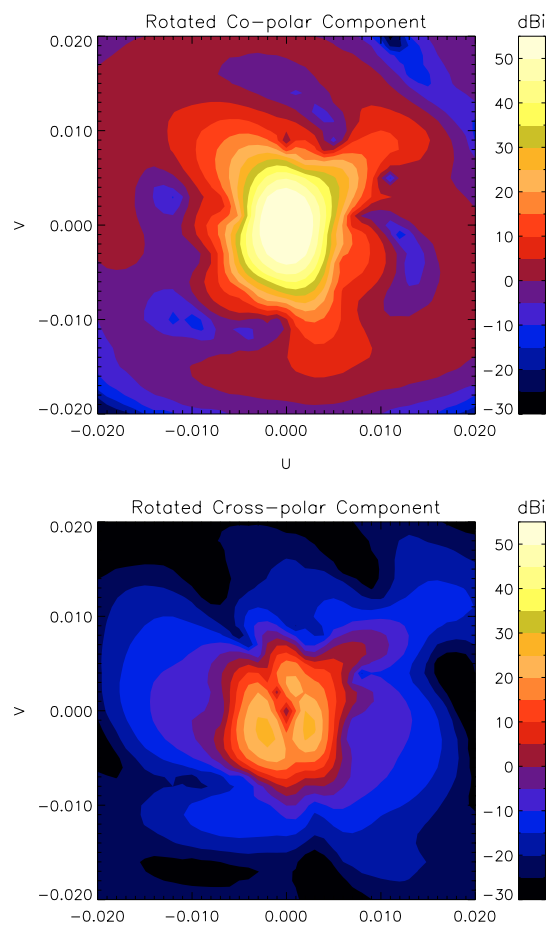

(c)

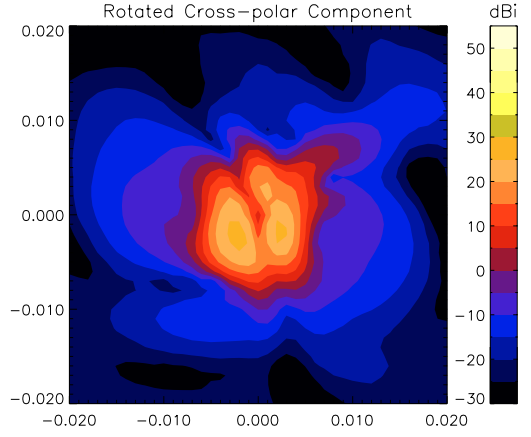

(b)

$\psi_{\mathrm{FP}}=0^{\circ}$
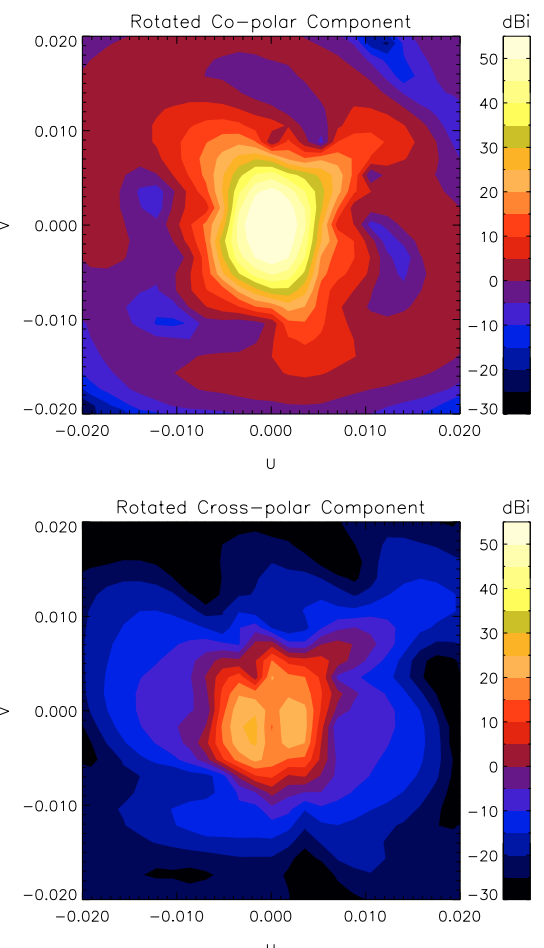

(d)

Fig. A.8. Same as Fig. A.7 (with $\left.|\theta|<1^{\circ}\right)$ for the $100 \mathrm{GHz}$ non-rotated feed $\left(\psi_{\mathrm{FP}}=0^{\circ}\right)$ at position 4 . In this figure the far field reference frame is rotated by b) $\phi_{\mathrm{XPD}}^{n r}=5.9^{\circ}$ (see Table 1$)$, c) $\phi_{\mathrm{XPD}}^{n r}=6.1^{\circ}$ (see Table 2) and d) $\phi_{\mathrm{XPD}}^{n r}=5.5^{\circ}$ (see Table 3 ). 
$100 \mathrm{GHz}$ Feed on Position 1
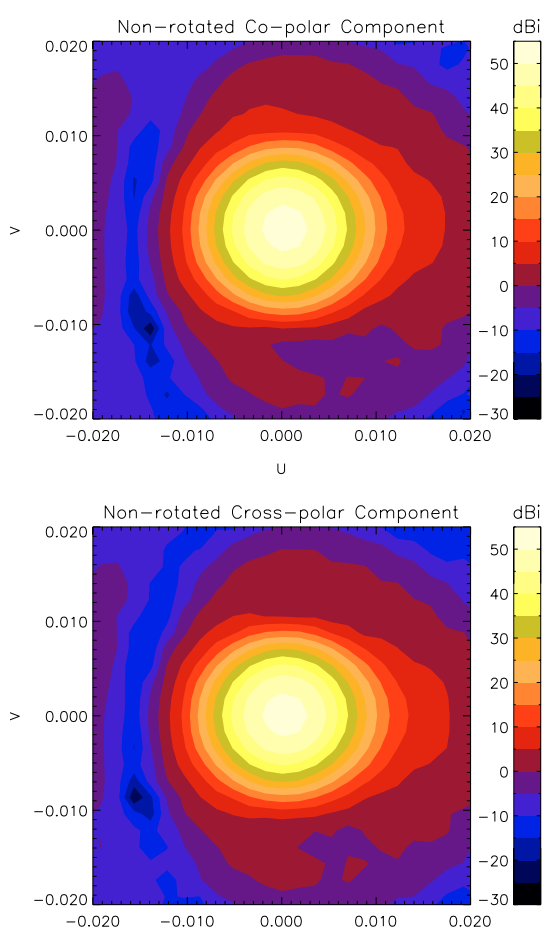

(a)
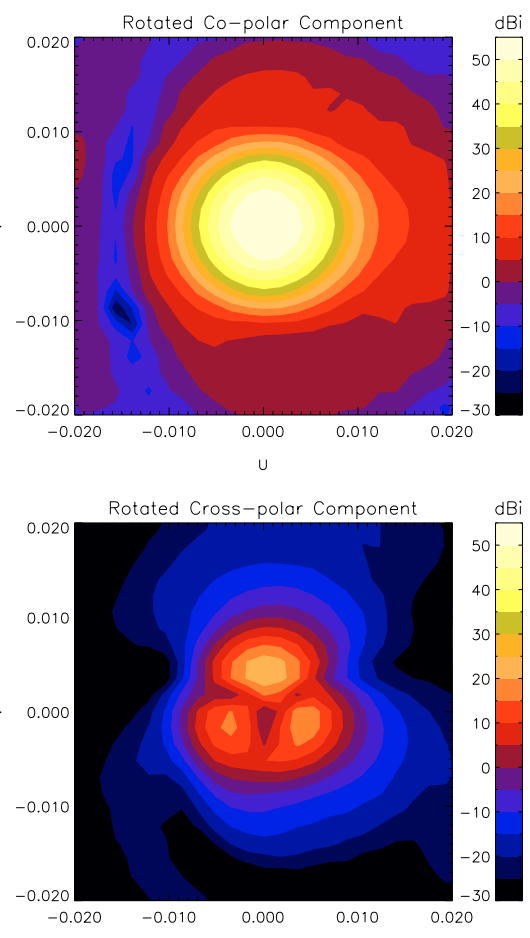

(b)

$30 \mathrm{GHz}$ Feed on Position 1
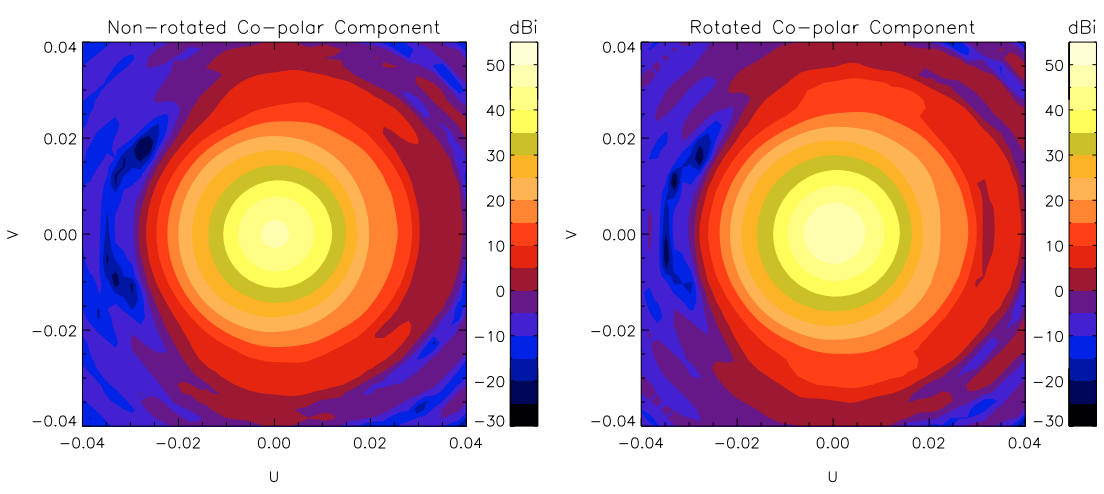

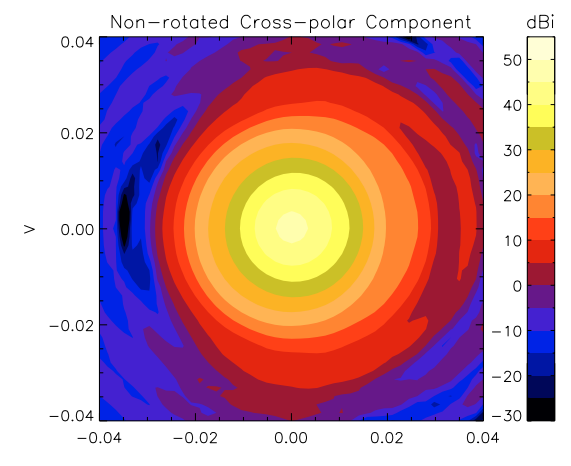

(c)

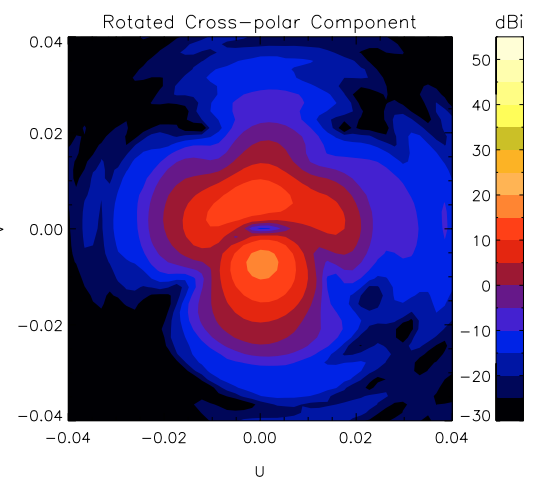

(d)

Fig. A.9. Same as Figs. A.7 and A.8 for the $100 \mathrm{GHz}$ feed rotated by $\psi_{\mathrm{FP}}=45^{\circ}$ (above) and the $30 \mathrm{GHz}$ feed rotated by $\psi_{\mathrm{FP}}=135^{\circ}$ (below), both at position 1. In this figure the far field reference frame is rotated by b) $\phi_{\mathrm{XPD}}=-44.5^{\circ}$ and $\mathbf{d}$ ) $\phi_{\mathrm{XPD}}=-134.5^{\circ}$ (see Table 3 ). 


\section{References}

Bennett, C. L., Kogut, A., Hinshaw, G., et al. 1994, ApJ, 436, 423

Carretti, E., Tascone, R., Corbiglioni, S., et al. 2001, New Astron., 6, 173

Couchot, F., Delabrouille, J., Kaplan, J., \& Revenu, B. 1999, A\&AS, 135,579

Crittenden, R., Bond, J. R., Davis, R. L., Efstathiou, G., \& Steinhardt P. J. 1993, Phys. Rev. Lett., 71, 324

Crittenden, R., Davis, R. L., \& Steinhardt, P. J. 1993, ApJ, 417, L13

de Oliveira-Costa,Tegmark, M., Zaldarriaga, M., et al. 2003, Phys. Rev. D, 67, 023003

Fosalba, P., Doré, O., \& Bouchet, F. R. 2002, Phys. Rev. D, 65, 63003

Hedman, M. M., Barkats, D., Gundersen, J. O., Staggs, S. T., \& Winstein, B. 2001, ApJ, 548, L111

Hu, W. 2000, ApJ, 529, 12

Kaplan, J., \& Delabrouille, J. 2001, in Astrophysical Polarized Backgrounds, ed. S. Cecchini, S. Cortiglioni, R. Sault, \& C. Sbarra, AIP Conf. Proc., 609, 209

Keating, B. G., O’Dell, C. W., de Oliveira-Costa, A., et al. 2001, ApJ, 560, L1

Kovac, J., Leitch, E. M., Pryke, C., \& Carlstrom, J. E. 2002, Nature, 420, 772

Kraus, J. D. 1982, Radio Astronomy (Ohio, USA)

Leahy, J. P., Yurchenko, V., Hastie, M. A., Bersanelli, M., \& Mandolesi, N. 2002, Astrophysical Polarized Backgrounds, held 9-12 October, 2001 in Bologna Italy, ed. S. Cecchini, S. Cortiglioni, R. Sault, \& C. Sbarra, AIP Conf. Proc., 609 (Melville, NY: American Institute of Physics), 215

Lewis, A., \& Bridle, S. 2002, Phys. Rev. D, 66, 103511

Lineweaver, C. H., Tenorio, L., Smoot, G. F., et al. 1996, ApJ, 470, 38

Ludwig, A. C. 1973, IEEE Trans. on Ant. and Prop., vol. AP-21, 116

Murphy, J. A., O'Sullivan, C., Henry, D., et al. 2000, Far IR Optics Design and Verification, Final Report of ESA TRP study contract number 13043/98/NL/NB (Phase 1). Note: the results of Phase 2 of the study, including the comparison of model to measurement, will be released in mid-2004.

Rees, M. J. 1968, ApJ, 153, L1

Scott, P. F., Carreira, P., Cleary, K., et al. 2003, MNRAS, in press

Tauber, J. 2000, The Extragalactic Infrared Background and its Cosmological Implications, IAU Symp., 204 (England: Manchester)

TICRA Engineering Consultants 1997, GRASP8 Software User Manual, Version 8.0.4 (Denmark: Copenhagen)

Yurchenko, V. 2001, AIP Conf. Proc. Experimental Cosmology at Millimeter Wavelengths: 2K1BC Workshop (Italy: Breuil-Cervinia), 616, 234

Zaldarriaga, M. 1997, Phys. Rev. D, 55, 1822

Zaldarriaga, M., Spergel, D. N., \& Seljak, U. 1997, ApJ, 488, 1 